\title{
A tale of two gradients: differences between the left and right hemispheres predict semantic cognition
}

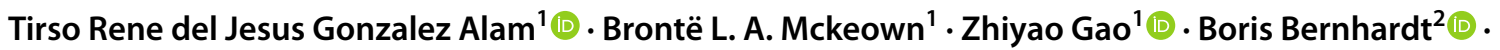

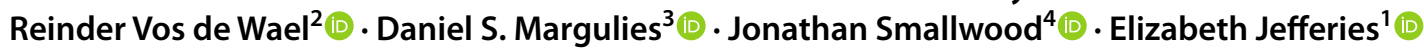

Received: 23 February 2021 / Accepted: 27 August 2021 / Published online: 12 September 2021

(c) The Author(s) 2021

\begin{abstract}
Decomposition of whole-brain functional connectivity patterns reveals a principal gradient that captures the separation of sensorimotor cortex from heteromodal regions in the default mode network (DMN). Functional homotopy is strongest in sensorimotor areas, and weakest in heteromodal cortices, suggesting there may be differences between the left and right hemispheres $(\mathrm{LH} / \mathrm{RH})$ in the principal gradient, especially towards its apex. This study characterised hemispheric differences in the position of large-scale cortical networks along the principal gradient, and their functional significance. We collected resting-state fMRI and semantic, working memory and non-verbal reasoning performance in $175+$ healthy volunteers. We then extracted the principal gradient of connectivity for each participant, tested which networks showed significant hemispheric differences on the gradient, and regressed participants' behavioural efficiency in tasks outside the scanner against interhemispheric gradient differences for each network. LH showed a higher overall principal gradient value, consistent with its role in heteromodal semantic cognition. One frontotemporal control subnetwork was linked to individual differences in semantic cognition: when it was nearer heteromodal DMN on the principal gradient in LH, participants showed more efficient semantic retrieval - and this network also showed a strong hemispheric difference in response to semantic demands but not working memory load in a separate study. In contrast, when a dorsal attention subnetwork was closer to the heteromodal end of the principal gradient in $\mathrm{RH}$, participants showed better visual reasoning. Lateralization of function may reflect differences in connectivity between control and heteromodal regions in LH, and attention and visual regions in RH.
\end{abstract}

Keywords Gradients $\cdot$ Intrinsic connectivity $\cdot$ Semantic cognition $\cdot$ Hemispheric specialization $\cdot$ Laterality $\cdot$ fMRI

\section{Introduction}

Contemporary accounts of brain organisation conceptualise cognition as reflecting interactions of large-scale networks of brain regions, organised in a systematic fashion along cortical gradients. These gradients capture similarities in connectivity patterns across disparate areas of the cortex

Tirso Rene del Jesus Gonzalez Alam

tirso.gonzalez.alam@york.ac.uk

Department of Psychology, University of York, York, UK

2 McConnell Brain Imaging Centre, Montreal Neurological Institute and Hospital, McGill University, Montreal, QC, Canada

3 Centre National de la Recherche Scientifique (CNRS) and Université de Paris, INCC UMR 8002, Paris, France

4 Queen's University, Kingston, ON, Canada
(Bressler and Menon 2010; Margulies et al. 2016; Medaglia et al. 2015; Paquola et al. 2018; Yeo et al. 2011). Cortical gradients provide a new tool for understanding patterns of hemispheric specialisation, since networks with lateralised connectivity will occupy different positions along these gradients in the left (LH) and right hemispheres (RH). This study exploits the potential of cortical gradients to uncover hemispheric differences in patterns of intrinsic connectivity, (i) by assessing the position of canonical networks in the left and right hemisphere along gradients derived bilaterally, and (ii) by examining the functional significance of these hemispheric differences for the highly left-lateralised domain of semantic cognition, compared with other cognitive domains (working memory (WM) and visual reasoning) that are expected to show different patterns of lateralisation.

The principal gradient, which explains the most variance in whole-brain decompositions of intrinsic connectivity, captures the separation between sensory-motor cortex and 
heteromodal Default Mode Network (DMN) (Huntenburg et al. 2018; Margulies et al. 2016). In this way, it relates to previously described cortical hierarchies that extract progressively more complex or heteromodal information from sensory inputs, or that maintain more abstract goals for action, in lateral and medial temporal lobes, and lateral and medial prefrontal cortex (Badre 2008; Badre and D'Esposito 2007; Bajada et al. 2017, 2019; Fuster 2001; Jackson et al. 2017, 2019; Koechlin et al. 2003; Petrides 2005; Thiebaut De Schotten et al. 2017). The principal gradient goes beyond these observations to explain why similar hierarchies occur in multiple brain regions. The principal gradient is correlated with physical distance along the cortical surface from primary systems, with the DMN falling at a maximum distance from sensory and motor systems in multiple locations across the cortex. Since DMN is a highly distributed network, with multiple nodes located in distant brain regions, the functional transitions captured by the principal gradient are repeated across the cortex, and these are seen in both hemispheres. The principal gradient also captures the sequence of networks found along the cortical surface-from DMN, through frontoparietal control networks, to attention networks (Dorsal and Ventral, DAN and VAN) and finally primary somatomotor and visual networks. A recent study showed that when gradient decomposition is performed for the two hemispheres separately, both hemispheres contain a similar (but not identical) principal gradient (Liang et al. 2021). However, the functional relevance of these similarities and differences between the left and right hemisphere has not been established.

Patterns of intrinsic connectivity tend to be highly symmetrical, with the strongest time-series correlations seen between homotopic regions that occupy the same position in the two hemispheres (Jo et al. 2012). However, symmetrical patterns of connectivity are weaker within heteromodal networks towards the DMN apex of the gradient (Raemaekers et al. 2018). These increasing asymmetries are related to structural connectivity: primary cortices are connected across the hemispheres through fast fibres of the corpus callosum, while heteromodal cortices are connected by slower fibres that show less homotopic connectivity (Stark et al. 2008). A recent study using large-scale novel meta-analytic and voxel mirroring methods confirmed that areas with less similar connectivity across hemispheres are associated with heteromodal functions, such as memory, language and executive control (Mancuso et al. 2019). Moreover, higher-order networks, including DMN, frontoparietal network (FPN) and dorsal attention network (DAN), show the highest degrees of interhemispheric differences in intrinsic connectivity (Karolis et al. 2019; Wang et al. 2014). These lateralised patterns of connectivity have functional significance, giving rise to lateralised functions like verbal semantics and other components of language (Joliot et al. 2016; Knecht et al. 2000) and aspects of attention (Bartolomeo and Seidel Malkinson 2019). For example, Gotts et al. (2013) identified that a 'segregation' mode of lateralisation in the left hemisphere (i.e., heightened intrinsic connectivity with other left hemisphere regions), conferred behavioural advantages in a verbal semantic task (vocabulary). In contrast, cross-hemisphere connections for the right hemisphere were related to better visual reasoning (block design). Given that segregated connectivity is also associated with higher-order heteromodal networks, we would expect this left hemisphere semantic pattern to involve lateralised connectivity at the heteromodal end of the gradient.

Previous studies have identified hemispheric differences in control networks, situated between DMN and sensorymotor cortex. In the left hemisphere, the frontoparietal control network couples preferentially to DMN and language regions, while in the right hemisphere, this network shows stronger connectivity to attentional regions (Wang et al. 2014). These findings suggest that control networks might be critical for the emergence of lateralised cognition. In line with this view, the most lateralised regions of the semantic network are associated with controlled semantic retrieval, as opposed to conceptual representation (Gonzalez Alam et al. 2019). Furthermore, the clustering of connectivity patterns within the FPN across hemispheres reveals a bipartite organisation, with one subnetwork showing more intrinsic connectivity to DMN, whilst the other shows more connectivity to DAN (Dixon et al. 2018). These subnetworks may support the capacity of the FPN to couple efficiently with the DAN and DMN, depending on the task (Niendam et al. 2012; Spreng et al. 2013; Vincent et al. 2008; Wang et al. 2014). These observations collectively give rise to the hypothesis that differences in network interactions between the hemispheres might be reflected in the location of control networks on the principal gradient, with left hemisphere control regions nearer to $\mathrm{DMN}$, and right hemisphere control areas nearer to the sensory-motor end of the gradient. In line with this view, Davey et al. (2016) suggested that leftlateralised semantic control processes reflect an interaction of heteromodal conceptual representations, associated with $\mathrm{DMN}$, and control processes that can promote the retrieval of currently-relevant aspects of knowledge, even when these are not dominant in long-term memory. Semantic cognition may be left lateralised because these DMN and control networks interact more strongly in the left hemisphere.

This study contrasted patterns of lateralisation for semantic cognition with working memory (Studies 1 and 2) and visual reasoning using matrix problems (Study 1). Since semantic cognition is thought to draw on left-lateralised interactions between DMN and control regions (Davey et al. 2016), working memory tasks provide an interesting contrast: increased working memory demands are expected to increase reliance on a bilateral multiple-demand network 
that supports executive demands across tasks (Duncan 2001, 2010; Fedorenko et al. 2013; Hugdahl et al. 2015), with the differential engagement of left and right hemispheres when verbal and spatial working memory tasks are compared (Emch et al. 2019; Hong et al. 2000). The multipledemand network is adjacent to but somewhat spatially distinct from the semantic control network (Davey et al. 2016; Gao et al. 2021; Jackson 2021), and its recruitment typically shows less lateralisation (Camilleri et al. 2018; Müller et al. 2015; Rottschy et al. 2012). Visual reasoning tasks are also expected to show a distinct pattern of lateralisation compared with semantic cognition. While semantic cognition is strongly left-lateralised, meta-analytic and patient evidence suggests a bilateral basis for a wide variety of reasoning tasks (Hobeika et al. 2016; Wertheim and Ragni 2018; Shin and Jeon 2021; Gläscher et al. 2010). Shin and Jeon (2021) found common bilateral activation in multiple demand cortex across inductive and deductive tasks, with stronger responses in the right hemisphere for more complex tasks. In some studies, matrix reasoning is more right-lateralised than analogical reasoning, consistent with this task's greater visual and spatial demands (Hobeika et al. 2016; but see also Wertheim and Ragni 2018). Visual reasoning tasks are expected to involve an interaction of control/attention networks with visual regions (Hearne et al. 2017), without strong engagement of memory processes in DMN; this pattern of network interaction might be bilateral or stronger in the right hemisphere. Structural equation modelling has shown that while executive and perceptual attention both contribute to the RAPM, executive control plays a larger role (Schweizer and Moosbrugger 2004; Ren et al. 2012; 2013). Although simple visual attention is thought to be rightlateralised (Kinsbourne 1987; Fink et al. 2000, 2001), the RAPM gives rise to bilateral responses with some evidence pointing to a right-lateralised bias under certain conditions (Prabhakaran et al. 1997; Bishop et al. 2008).

Study 1 examined the organisation of the principal gradient across the left and right human cerebral hemispheres in participants who took part in a resting-state scan $(N=253)$ and behavioural tasks in a separate session $(N=175)$. We considered how individual differences in semantic cognition related to the position of large-scale networks on the principal gradient of intrinsic connectivity defined by Margulies et al. (2016), in the left versus right hemispheres, deriving a hemispheric difference gradient score per network for each participant. The semantic component that we examined was derived from a wide variety of semantic tasks, and is likely to reflect the capacity to access relevant conceptual knowledge in different contexts. We would expect this component to be left-lateralised as all elements of the semantic cognition network appear to show a left-hemisphere bias in metaanalytic evidence (the map for the term 'semantic cognition' from Neurosynth, for example, is highly left-lateralised), with the possible exception of the anterior temporal lobe (Rice et al. 2015a, b; Jackson et al. 2017; Gonzalez Alam et al. 2019). These effects for semantic judgements were compared with Raven's Advanced Progressive Matrices (RAPM), a measure of non-verbal reasoning, and Digit Span, a measure of verbal working memory, allowing us to determine whether hemispheric differences on the principal gradient are related in distinct ways to left-lateralised semantic cognition (Gonzalez Alam et al. 2019; Jackson 2021; Noonan et al. 2013), compared with other types of demanding cognition. Using similar methods, Mckeown et al. (2020) found associations between individual differences in gradient values and patterns of spontaneous thought, suggesting that variation in gradient organisation is reflected in people's cognition and experience.

Having established that individual differences in semantic cognition were associated with the magnitude of hemispheric differences on the principal gradient in a specific control network in Study 1, we examined how semantic and non-semantic task demands modulated activation within this lateralised control network in Study 2. We re-analysed fMRI data examining parametric manipulations of difficulty in semantic and verbal working memory tasks (Gao et al. 2021). Controlled semantic retrieval demands were varied by presenting word pairs that were strongly or more weakly associated: previous studies have shown greater recruitment of the left-lateralised semantic control network when participants are required to identify weak associations that are not dominant within the semantic store (Jackson 2021; Noonan et al. 2013). Semantic control demands were compared with the effects of working memory load, since higher working memory demands increase the recruitment of bilateral multiple-demand cortex that is partially distinct from the semantic control network (Fedorenko et al. 2013). In this way, we assessed whether hemispheric differences in the position of large-scale networks on the principal gradient of intrinsic connectivity at rest corresponded with hemispheric differences in the recruitment of these networks during task performance.

\section{Methods}

\section{Study 1}

\section{Participants}

Two hundred and seventy-seven healthy participants were recruited from the University of York. Written informed consent was obtained for all participants and the study was approved by the York Neuroimaging Centre Ethics Committee. The participants were right-handed, native English speakers with normal/corrected vision. None of 
them had a history of psychiatric or neurological illness, severe claustrophobia, drug use that could alter cognitive functioning, or pregnancy. Twenty-four participants were excluded from fMRI analyses; two due to technical issues during the neuroimaging data acquisition, one due to a data processing error and twenty-one for excessive movement during the scan (Power et al. 2014; mean framewise displacement $>0.3 \mathrm{~mm}$ and/or more than $15 \%$ of their data affected by motion), resulting in a final cohort of $N=253$ ( 169 females, mean \pm SD age $=20.7 \pm 2.4$ years). A subset of 175 of these participants also completed a semantic relatedness judgement task, a digit span task and Raven's Progressive Matrices (along with other behavioural tasks outside the scope of this study), in a separate session. While the current analysis of hemispheric gradient differences is novel, this data has been used in previous studies to examine the neural basis of memory and mind-wandering, including region-ofinterest based connectivity analysis and cortical thickness investigations (Evans et al. 2020; Gonzalez Alam et al. 2018, 2019, 2021; Karapanagiotidis et al. 2017; Poerio et al. 2017; Sormaz et al. 2018; Turnbull et al. 2018; Vatansever et al. 2017; Wang et al. 2018a, b).

\section{Procedure}

All participants underwent a 9 min resting-state fMRI scan. During the scan, they were instructed to passively view a fixation cross and not to think of anything in particular. Immediately following the scan, they completed a 25 -item experience-sampling questionnaire while still in the scanner; these data have been reported in Karapanagiotidis et al. (2020) and Mckeown et al. (2020). In two subsequent sessions, participants completed a battery of semantic and other cognitive tasks. These sessions included personality and wellbeing scales, and measures of perception, episodic memory, cognitive control and mind-wandering, which were beyond the scope of this investigation.

\section{Materials}

Given our focus is on the lateralisation of semantic cognition, we selected a semantic judgement test battery for analysis. We contrasted the pattern for left-lateralised semantic cognition with two non-semantic tasks expected to have a different pattern of lateralisation - Raven's progressive matrices (Raven et al. 1994), involving visual reasoning, and forwards digit span adapted from the Weschler Adult Intelligence Scale (WAIS; Wechsler 1955). Raven's progressive matrices engages bilateral visual and attentional control processes that have a right-hemisphere bias (Bishop et al. 2008; Corbetta et al. 2008; Haier et al. 1988; Prabhakaran et al. 1997; Ren et al. 2012; Schweizer and Moosbrugger 2004), while the digit span task engages left as well as right-lateralised aspects of multiple-demand cortex (Fedorenko et al. 2013; Hugdahl et al. 2015). This task comparison can therefore establish whether any hemispheric differences in gradient values relating to semantic cognition are specific to the semantic domain, or are found more generally for demanding tasks.

Semantic task Participants performed semantic relatedness judgements that manipulated modality (words/pictures) and strength of association (weak/strong associates; see Fig. 1). The task employed a three-alternative forcedchoice design: participants matched a probe stimulus on the screen with one of three possible targets, and pressed buttons to indicate their choice. Each trial consisted of a centrally presented probe preceded by a target and two unrelated distractors, which were targets in other trials. Trials started with a blank screen for $500 \mathrm{~ms}$. The three response options were subsequently presented at the bottom of the screen for $900 \mathrm{~ms}$ (aligned horizontally, with the target in each location equally often). Finally, the probe was presented at the top of the screen. The probe and choices remained visible until the participant responded, or for a maximum of $3 \mathrm{~s}$. Both response time (RT) and accuracy were recorded, and an efficiency score was calculated for each participant in each condition by dividing response times by accuracy, and multiplying that ratio by -1 , so that higher scores reflected better performance. This approach allowed us to control for speed-accuracy trade-offs when assessing associations with intrinsic connectivity and avoids the inflation of Type 1 errors that would result from running parallel analyses for accuracy and RT separately. The Supplementary Materials describe assessments of the suitability of the data for response efficiency analysis (see Supplementary Analysis: assumption check for efficiency scores).

The stimuli employed in the tasks were selected from a larger set of words and photographs used in previous experiments (Davey et al. 2015; Krieger-Redwood et al. 2015). The pictures were coloured photographs collected from the internet and re-sized to fit the trial structure (200 pixels, $72 \mathrm{dpi}$ ). All the coloured pictures and words were rated for familiarity using 7-point Likert scales, and imageability ( $>500)$ from the MRC psycholinguistic database (Coltheart 1981; Wilson 1988). Lexical frequency for the words was obtained by the SUBTLEX-UK database (van Heuven et al. 2014) to allow matching on psycholinguistic properties. The strength of association between probe-target pairs was assessed using a 7-point Likert scale and differed significantly between conditions. There were no differences between strong and weak associations in word length, familiarity, imageability or lexical frequency. The order of trials within the blocks (conditions) was randomized across subjects. Each block contained 60 trials. The presentation of the blocks was interleaved. 

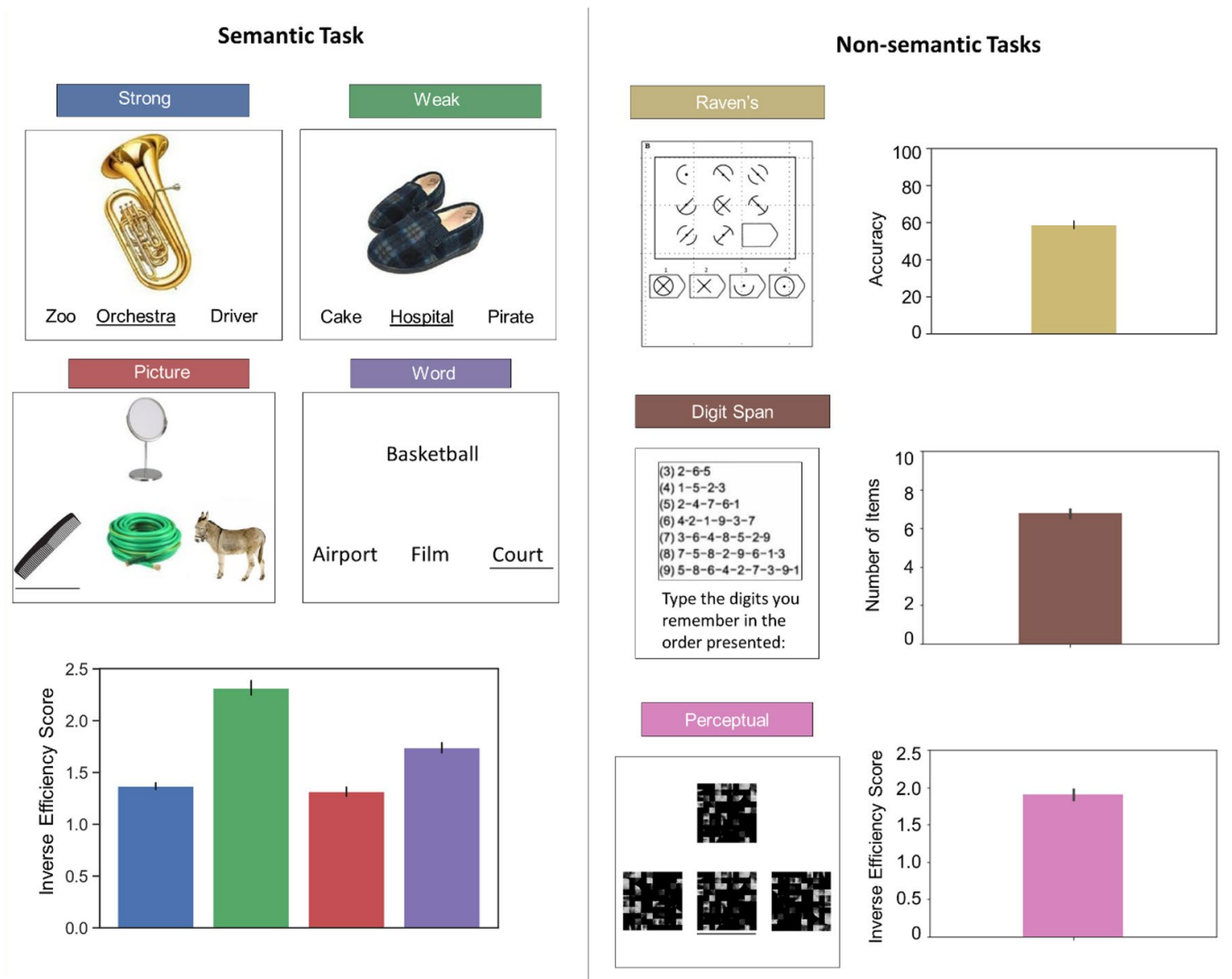

Fig. 1 Illustration of the semantic (left panel) and non-semantic (right panel) tasks employed in this study. The bar plots are colour coded to match the examples depicting each condition of the tasks (i.e., the blue bar in the left panel corresponds to the 'strong' condition). Performance in the semantic and perceptual tasks are depicted here using inverse efficiency scores, in which larger numbers correspond

Semantic dimensionality reduction Given that efficiency scores were correlated across the conditions of the task, we performed data-driven dimensionality reduction, which revealed a single semantic factor in the relatedness judgement task. PCA with varimax rotation yielded one single factor with Kaiser's criterion above 1, explaining $75 \%$ of the variance (see Supplementary Fig. 9). Each participant's efficiency scores in the four tasks were therefore summarised using a single score reflecting the single factor loading, which was carried forward into regression analysis after $z$-scoring and imputing any outlier above \pm 2.5 with the mean. This metric is likely to reflect individual differences in general semantic performance, since it loaded on both verbal and pictorial semantic tasks, and conditions requiring little semantic control (i.e., strong associations) as well as greater semantic control (i.e., weak associations). The component scores are likely to reflect the global efficiency of conceptual retrieval resulting from both the strength of het-

to worse performance. Elsewhere, efficiency scores were multiplied by -1 , for interpretability. Digit span is expressed as a maximum number of items recalled, and Raven's as accuracy (number of problems solved out of a maximum of 36). Error bars depict $95 \%$ confidence intervals

eromodal semantic representations as well as the capacity to recruit control processes to support semantic cognition when needed. Summary measures of the behavioural data in each condition of the semantic task can be consulted in Supplementary Materials (Tables 4 and 5).

Raven's advanced progressive matrices The Ravens Advanced Progressive Matrices (Raven et al. 1994) is a measure of non-verbal reasoning that requires participants to identify meaningless visual patterns. The progressive matrices task included 36 questions, preceded by two practice trials. During the practice phase, participants were given feedback and task training, with no feedback for the reminder of the trials. For each problem, a set of 9 tiles (in a $3 \times 3$ design) were shown on the screen. All but one tile contained a pattern. At the bottom of the screen were 4 additional patterned tiles. Participants were required to select which tile would complete the pattern (see Fig. 1). Participants were given 
20 min to complete as many problems as they could, and the problems got progressively more difficult.

Digit span For the Digit Span Task, we used the stimuli and the scoring procedure described in the WAIS battery (Wechsler 1955), IV edition. For each trial, audio files of each digit were played in the sequential order reported in the WAIS battery. The instructions were presented at the beginning of each block asking participants to listen to the sequence of numbers and type them in the same order (see Fig. 1).

Perceptual figure matching task The semantic, working memory and visual reasoning tasks used in this study varied in the number of response options, time available to respond and layout of presentation. Therefore, we included a behavioural covariate in the regression analysis that captured performance on a non-semantic figure matching task that was matched to the semantic battery in terms of the number of perceptual inputs, decision-making format, and mode of response. In this figure matching task, participants decided whether two scrambled images were mirror images of each other. The stimuli were 60 pixelated and scrambled blackand-white photographs of faces (Krieger-Redwood et al. 2013). Participants were asked to select the target that was identical to the probe; the distracters were the same images rotated by $180^{\circ}$ or $270^{\circ}$. The task was split in two blocks of 30 trials each (see Fig. 1).

\section{Neuroimaging}

The MRI data acquisition and pre-processing steps reported in this paper are identical to the steps reported in Karapanagiotidis et al. (2020), and the dimension reduction steps are identical to the ones reported in Mckeown et al. (2020), as reproduced in the sections below.

MRI data acquisition MRI data were acquired on a GE 3T Signa Excite HDx MRI scanner, equipped with an eightchannel phase-array head coil at York Neuroimaging Centre, University of York. For each participant, we acquired a sagittal isotropic 3D fast spoiled gradient-recalled echo T1-weighted structural scan $(\mathrm{TR}=7.8 \mathrm{~ms}, \mathrm{TE}=$ minimum full, flip angle $=20^{\circ}$, matrix $=256 \times 256$, voxel size $=1.13 \times 1.13 \times 1 \mathrm{~mm}^{3}$, FOV $=289 \times 289 \mathrm{~mm}^{2}$ ). Resting-state fMRI data based on blood oxygen level-dependent contrast images with fat saturation were acquired using a gradient single-shot echo-planar imaging sequence $(\mathrm{TE}=$ minimum full $(\approx 19 \mathrm{~ms})$, flip angle $=90^{\circ}$, matrix $=64 \times 64$, FOV $=192 \times 192 \mathrm{~mm}^{2}$, voxel size $=3 \times 3 \times 3 \mathrm{~mm}^{3}$, $\mathrm{TR}=3000 \mathrm{~ms}, 60$ axial slices with no gap and slice thickness of $3 \mathrm{~mm}$ ). Scan duration was 9 min which allowed us to collect 180 whole-brain volumes.
MRI data pre-processing fMRI data pre-processing was performed using SPM12 (http://www.fil.ion.ucl.ac.uk/ spm) and the CONN toolbox (v.18b) (https://www.nitrc. org/projects/conn) (Whitfield-Gabrieli and Nieto-Castanon 2012) implemented in Matlab (R2018a) (https://uk.mathw orks.com/products/matlab). Pre-processing steps followed CONN's default pipeline and included motion estimation and correction by volume realignment using a six-parameter rigid body transformation, slice-time correction, and simultaneous grey matter (GM), white matter and cerebrospinal fluid (CSF) segmentation and normalisation to MNI152 stereotactic space (2 $\mathrm{mm}$ isotropic) of both functional and structural data. Following pre-processing, the following potential confounders were statistically controlled for: 6 motion parameters calculated at the previous step and their 1st and 2nd order derivatives, volumes with excessive movement (motion greater than $0.5 \mathrm{~mm}$ and global signal changes larger than $z=3$ ), linear drifts, and five principal components of the signal from white matter and CSF using the CompCor approach (Behzadi et al. 2007). Finally, data were band-pass filtered between 0.01 and $0.1 \mathrm{~Hz}$. No global signal regression was performed.

Gradient analysis We obtained each participant's gradient values for the first principal gradient (Margulies et al. 2016) following the methods described in Mckeown et al. (2020). Following pre-processing, the functional time-series from 400 ROIs based on the Schaefer parcellation (Schaefer et al. 2018; Yeo et al. 2011) were extracted for each individual. A connectivity matrix was then calculated using Pearson correlation resulting in a $400 \times 400$ connectivity matrix for each participant. These individual connectivity matrices were subjected to a Fisher $Z$ transform prior to averaging, and then used to calculate a group-averaged connectivity matrix. The BrainSpace Toolbox (Vos de Wael et al. 2020) was used to extract ten group-level gradients from the group-averaged connectivity matrix (dimension reduction technique $=$ diffusion embedding, kernel $=$ normalized angle, sparsity $=0.9$ ). This study was primarily focussed on the first gradient, which has well-described functional associations relevant to previous lateralisation findings; however, we extracted ten gradients to maximize the degree of fit between the groupaveraged gradients and the individual-level first gradient (this method is justified by the analysis in Supplementary Table S2, which shows a higher degree of fit with the canonical group-level gradients established by Margulies et al. (2016) when extracting ten gradients compared with three). The variance explained by each group-averaged gradient is provided in Supplementary Fig. 6.

The group-level gradient solutions were aligned using Procrustes rotation to a subsample of the HCP dataset $(N=217,122$ women, mean \pm SD age $=28.5 \pm 3.7 \mathrm{y}$; for further details about subject selection and the benefits of 
this gradient alignment step, see Vos De Wael et al. 2018). The Procrustes rotation improves correspondence between the canonical gradients described by Margulies et al. (2016) and the group-level gradient solutions by rotating, translating and optionally scaling the group-level matrix to achieve maximum similarity with the target matrix minimizing the sum of squared differences. Procrustes rotation was chosen, as opposed to joint embedding, as it preserves the overall shape of the gradients (Vos de Wael et al. 2020). The first three group-averaged gradients, with and without alignment to the HCP data, are shown in Supplementary Fig. 7. To demonstrate the benefits of this alignment step, we calculated the similarity using Spearman Rank correlation between the first five aligned and unaligned group-level gradients to the first five gradients reported in Margulies et al. (2016), which were calculated using 820 participants over an hour-long resting-state scan. Alignment improved the stability of the group-level gradient templates by maximising the comparability of the solutions to those from the existing literature (i.e., Margulies et al. 2016; see our Supplementary Table S3).

Using identical parameters, individual-level gradients were then calculated for each individual using their $400 \times 400$ connectivity matrix. These individual-level gradient maps were aligned to the group-level gradient maps using Procrustes rotation to improve the comparison between the group-level gradients and individual-level gradients $(N$ iterations $=10)$. This analysis resulted in ten group-level gradients and ten individual-level gradients for each participant explaining maximal whole-brain connectivity variance in descending order. Procrustes rotation was also used to address the reordering of gradient components (since sometimes gradients at the participant level are not in the same order as the canonical gradients at the group level). As stated above, this report focuses on the principal gradient (with supplementary analyses for Gradient 2), since this gradient captures the sequence of largescale networks on the cortical surface. To demonstrate the variability of individual-level gradients, Supplementary Fig. 8 shows the highest, lowest, and median similarity gradient maps for the principal gradient.

Hemispheric difference analysis As a first step for our analysis of interest, we obtained group averages of the principal gradient for each of the 400 parcels per participant (top row of Fig. 3). Since these parcels do not necessarily share homotopes across hemispheres, for the hemispheric difference analyses we summarised these values by averaging, for each participant, the parcels corresponding to each of the 17 networks described by Yeo et al. (2011). We will refer to these two levels of analyses as 'parcel level' and 'network level', respectively.
Next, we examined hemispheric differences across the 17 Yeo Network parcellation. We normalised each parcel's principal gradient value within each participant using a minimum-maximum normalisation (0-100) before computing their network's average, such that networks toward the lower end of the principal gradient have values closer to 0 , and networks towards DMN have values close to 100 (the middle row of Fig. 3 shows the group average per network; the organisation of these networks are depicted in the bottom row of Fig. 3). We tested for hemispheric differences in the global gradient value by averaging all gradient values across the 17 Yeo networks within each hemisphere separately for each participant in the sample and comparing these means using a paired $t$ test (left hemisphere vs right hemisphere).

Our next step involved subtracting the average of each right hemisphere network from its homotope in the left hemisphere, for each participant (we $z$-scored the results to produce a group difference map highlighting the networks with the most extreme differences shown in Fig. 4). We then performed a two-way repeated-measures ANOVA, using Hemisphere and Network as between-subject factors, to test for hemispheric differences at the network level. Having obtained significant main effects and an interaction, we conducted post-hoc non-parametric permutation testing with 5000 bootstrapped samples to compute the probability of obtaining a difference of gradient means across hemispheres as extreme as that empirically observed for each network by chance (Fig. 5). The non-parametric $p$ values of these post-hoc tests were Bonferroni-corrected at an alpha $=0.05$ for 17 multiple comparisons to guard against Type 1 errors. We only included those networks that showed significant hemispheric differences in the subsequent analyses.

Behavioural regressions To examine whether hemispheric differences on the principal gradient across networks had behavioural consequences, we performed regression analyses relating participants' performance outside the scanner on semantic and non-semantic tasks (working memory and visual reasoning) to the difference in principal gradient values across the hemispheres for each significant network. We entered each participant's semantic factor loading as an Explanatory Variable (EV) into an Ordinary Least Squares (OLS) regression, using hemispheric difference scores on the principal gradient for each network as the dependent variable. The semantic factor was added together with participants' $z$-scored performance on Raven's matrices, digit span and perceptual judgements matched superficially to the semantic tasks. All four of these task EVs were entered together for each regression model. An additional analysis examined individual differences in variation between the conditions of the semantic task, to investigate the effect of modality (pictures versus words) and difficulty (weak versus strong associates) but no patterns of lateralisation along the 
principal gradient were related to these subtler aspects of behaviour.

\section{Supplementary analysis of the second gradient}

While our main focus is on the principal gradient, we provide a supplementary analysis of the second gradient as described in Margulies et al. (2016), which captures the difference in connectivity between visual and motor networks. We show the means per hemisphere for Gradient 2, along with group means for each of the 400 parcels from Schaefer et al. (2018), and the 17 networks from Yeo et al. (2011). In this analysis, we characterise hemispheric differences per network for this gradient. Lastly, we provide bootstrapping analyses of the left versus right hemisphere network differences and establish which networks survive correction for multiple comparisons. All of these analyses follow the methods described above, with the results presented in Supplementary Analysis: Gradient 2, Supplementary Figs. 2-5.

\section{Study 2}

\section{Parametric manipulations of semantic control and working memory load}

Previous research has shown the controlled retrieval of semantic information elicits activation within a highly leftlateralised semantic control network (Gonzalez Alam et al. 2019; Jackson 2021; Noonan et al. 2013), which is at least partially distinct from the bilateral multiple demand network that supports other aspects of cognitive control (Davey et al. 2016; Gao et al. 2021; Gonzalez Alam et al. 2018; KriegerRedwood et al. 2015). Having established in Study 1 that individual differences in semantic cognition were associated with the magnitude of hemispheric differences on the principal gradient within a particular control network (ControlB), we then examined how semantic and non-semantic task demands modulate activation within this control network in Study 2.

We re-analysed an fMRI dataset (Gao et al. 2021) examining the effects of semantic control demands (via a parametric manipulation of strength of association) and verbal working memory load (using a parametric manipulation of the number of items to be maintained). In this new analysis, we extracted the effect of these parametric regressors on the BOLD response in left- and right-hemisphere components of large-scale networks that showed behavioural associations with lateralisation effects on the principal gradient in Study 1. Previous research has shown that retrieving semantic links between more weakly-related words elicits strong engagement of the left-lateralised semantic control network (Jackson 2021; Noonan et al. 2013). In contrast, higher loads in WM tasks are associated with greater responses within the
Multiple Demand Network, particularly within the left hemisphere for verbal materials (Emch et al. 2019; Fedorenko et al. 2013). Our analysis, therefore, allowed us to compare the effects of task demands in domains associated with distinct control networks, with potentially different patterns of lateralisation. This experiment did not include a visual reasoning task comparable to Ravens Advanced Progressive Matrices, and consequently our focus was on comparing leftlateralised semantic cognition with another verbal yet nonsemantic task. The tasks were broadly matched in terms of input processing and motor responses; however, the way in which control demands were manipulated was not identical across these tasks (i.e., difficulty of retrieval versus amount of information to be maintained).

We predicted a lateralised response to semantic but not non-semantic control demands specifically for networks in which individual differences in semantic cognition were associated with hemispheric differences in principal gradient values-i.e., a stronger response to semantic control than to working memory demands in the left but not right hemisphere within the Control-B network.

\section{Participants}

As reported in Gao et al. (2021), a group of 32 young healthy participants aged $19-35$ (mean age $=21.97 \pm 3.47$ years; 19 females) was recruited from the University of York. They were all right-handed, native English speakers, with normal or corrected-to-normal vision and no history of psychiatric or neurological illness. The study was approved by the Research Ethics Committee of the York Neuroimaging Centre. All volunteers provided informed written consent and received monetary compensation or course credit for their participation. The data from one task was excluded for four participants due to head motion, and one additional WM dataset was excluded due to errors in recording the responses. The final sample included 28 participants for the semantic task and 27 participants for the WM task, with 26 participants completing both tasks.

\section{Materials and procedure}

The materials and procedure for this experiment (as well as the results reported in Sect. Study 2.) are described fully in Gao et al. (2021). We summarise below the aspects that are relevant to the present study.

Semantic task In the on-line semantic task in fMRI, participants had to judge whether pairs of words were semantically related or unrelated. The stimuli were 192 English concrete noun word-pairs (abstract words and words drawn from the same taxonomic category were excluded, so that only thematic links were evaluated). The stimuli had parametrically 
varying degrees of thematic relatedness depending on their frequency of co-occurrence. The degree of relatedness was quantified using distributed representations of word meanings obtained from the word2vec neural network, trained on the 100 billion-word Google News dataset (Mikolov et al. 2013), defining the strength of the semantic relationship between pairs of words using the cosine similarity method. The stimulus set was manipulated so there was a parametric continuum of relatedness, from 'not related at all' to 'strongly related'. Since the degree of relatedness was continuous, there were no clear 'correct' or 'incorrect' answers; instead, the trials were sorted according to whether participants judged each trial to be related or unrelated based on their own experience. Difficulty was then estimated by binning the stimuli into 5 categories for related and unrelated trials separately according to their word2vec score for each individual participant (since pairs judged to be related varied across participants). For trials judged to be related, a lower word2vec score was associated with increased difficulty (since establishing a semantic link for less strongly related items is harder); conversely, for trials judged to be unrelated, a higher word2vec score increased difficulty (since rejecting a relationship between associated words is harder). Each trial began with a visually presented word $(1.5 \mathrm{~s})$, followed by a central fixation $(1.5 \mathrm{~s})$, then the second word $(1.5 \mathrm{~s})$, followed by a $3 \mathrm{~s}$ response period where participants indicated whether the word was related or not pressing one of two buttons with their right hand. Participants performed 4 runs of this task, each lasting $7.3 \mathrm{~min}$. Both response time and choice were recorded.

Word2vec has been shown to predict human behaviour better than other approaches such as latent semantic analysis (Pereira et al. 2016). Previous research has shown that semantic distance, as measured by word $2 \mathrm{vec}$, is negatively correlated with the strength of activation in the semantic control network: weakly-related trials require more controlled retrieval to identify a semantic link (Hoffman 2018; Teige et al. 2019), engaging well-defined regions of the semantic control network, including left inferior frontal gyrus (Zhang et al. 2021); this allows us to use word2vec scores as a proxy for semantic control demands (Badre et al. 2005; Teige et al. 2019; Wagner et al. 2001).

Working memory task Non-semantic control demands were manipulated in a verbal working memory task, using a parametric manipulation of the number of items participants had to maintain in memory. This task had a similar structure and method of presentation to the semantic task; each trial began with a letter string ( 3 to 7 letters) presented at the centre of the screen (for $1.5 \mathrm{~s}$ ), followed by a fixation (for $1.5 \mathrm{~s}$ ). Participants were asked to remember these letters. Next, two letters were shown on the screen (for $1.5 \mathrm{~s}$ ) and participants judged whether both of them had been presented in the let- ter string by pressing one of two buttons (with this decision phase presented for $3 \mathrm{~s}$ ). Participants were told that the order of the letters did not matter. The working memory load was manipulated by varying the number of letters memorised in each trial; there were five levels of load from 3 to 7 letters (to match the 5 levels of word2vec in the semantic task). Both response time and accuracy were recorded. Participants completed 3 runs, each containing 40 trials and lasting for $6.1 \mathrm{~min}$.

\section{Analysis}

The univariate analysis of these parametric manipulations yielded effect maps for semantic control and working memory demands, reported in Gao et al. (2021), which we employed in a ROI analysis to examine which networks showed significant task differences across hemispheres. For the participant level analysis, we binarised the Yeo network maps that showed significant behavioural associations in Study 1 and used them as ROI masks to extract the percent signal change value for each of the 26 participants separately in the left and right hemispheres for each condition of the task (i.e., related and unrelated semantic judgements, and working memory) using the featquery tool in FSL 6. We entered these values for each participant into separate repeated-measures ANOVAs for each network, examining 'Hemisphere' and 'Condition' as within-subjects factors.

\section{Results}

\section{Study 1}

\section{Gradient values for the Schaeffer parcellation in left and right hemispheres}

Our first analysis step revealed a global mean difference on the principal gradient (see Fig. 2), with higher values in the left hemisphere (paired samples $t$ test: $t(252)=18.38$, $p<0.001$ ); the presence of higher global mean values in the left compared to the right hemisphere was observed in $87.7 \%$ of our sample (see the scatterplot in Fig. 2). Participants' left and right hemisphere mean gradient values were very highly correlated (Pearson's $r=0.9, p<0.001$ ), despite this global difference.

As expected, given our gradient alignment methods, the gradient decomposition of our 253-participant sample showed a principal gradient very similar to the one reported by Margulies et al. (2016), both at the parcel level (Fig. 3, top row) and at the network level (Fig. 3, middle row); see also Mckeown et al. (2020). 

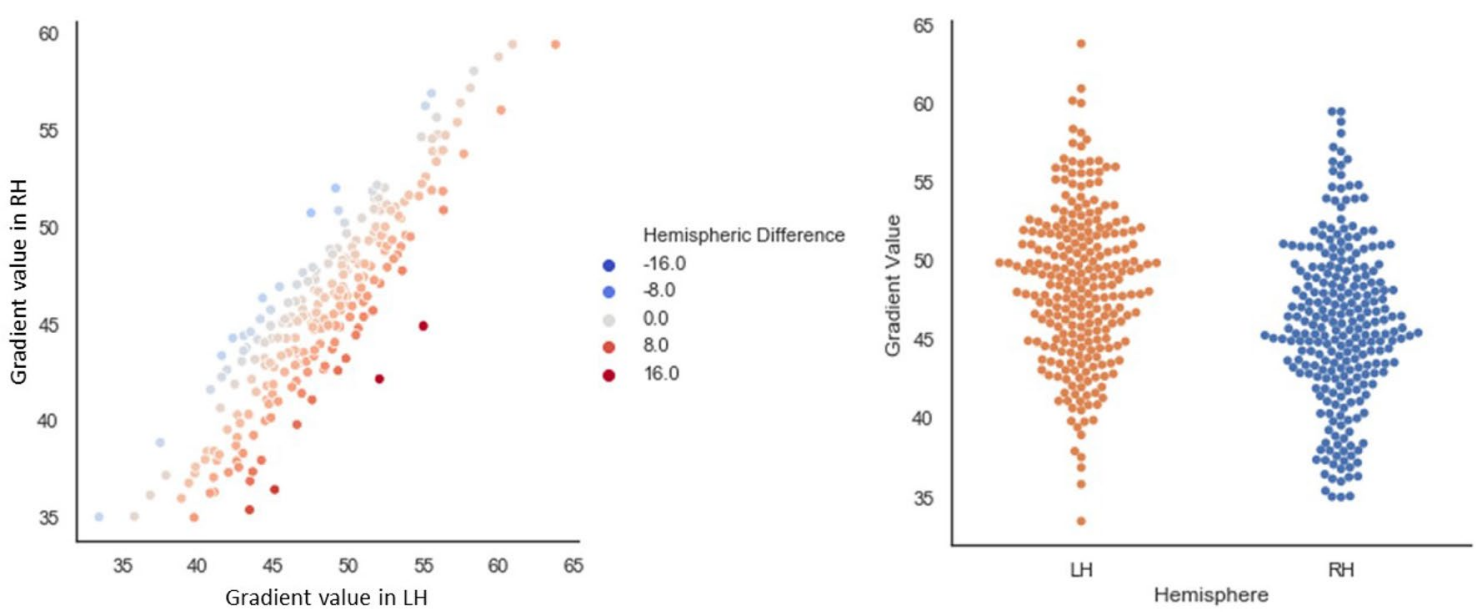

Fig. 2 The left panel depicts a linear relationship in our sample's mean left and right hemisphere values on the principal gradient. The 'Hemispheric Difference' legend of the scatterplot depicts the result of subtracting the LH-RH mean gradient loadings for the whole hemisphere per participant. Positive values reflect closer proximity to the heteromodal end of the gradient in LH. Negative values reflect closer proximity to the heteromodal end of the gradient in RH. The right panel depicts the distributions of mean global hemispheric values per participant in our sample. In both plots, each dot represents one participant. The scale on both plots indicates values on the principal gradient, which were re-scaled to range from 0 to 100

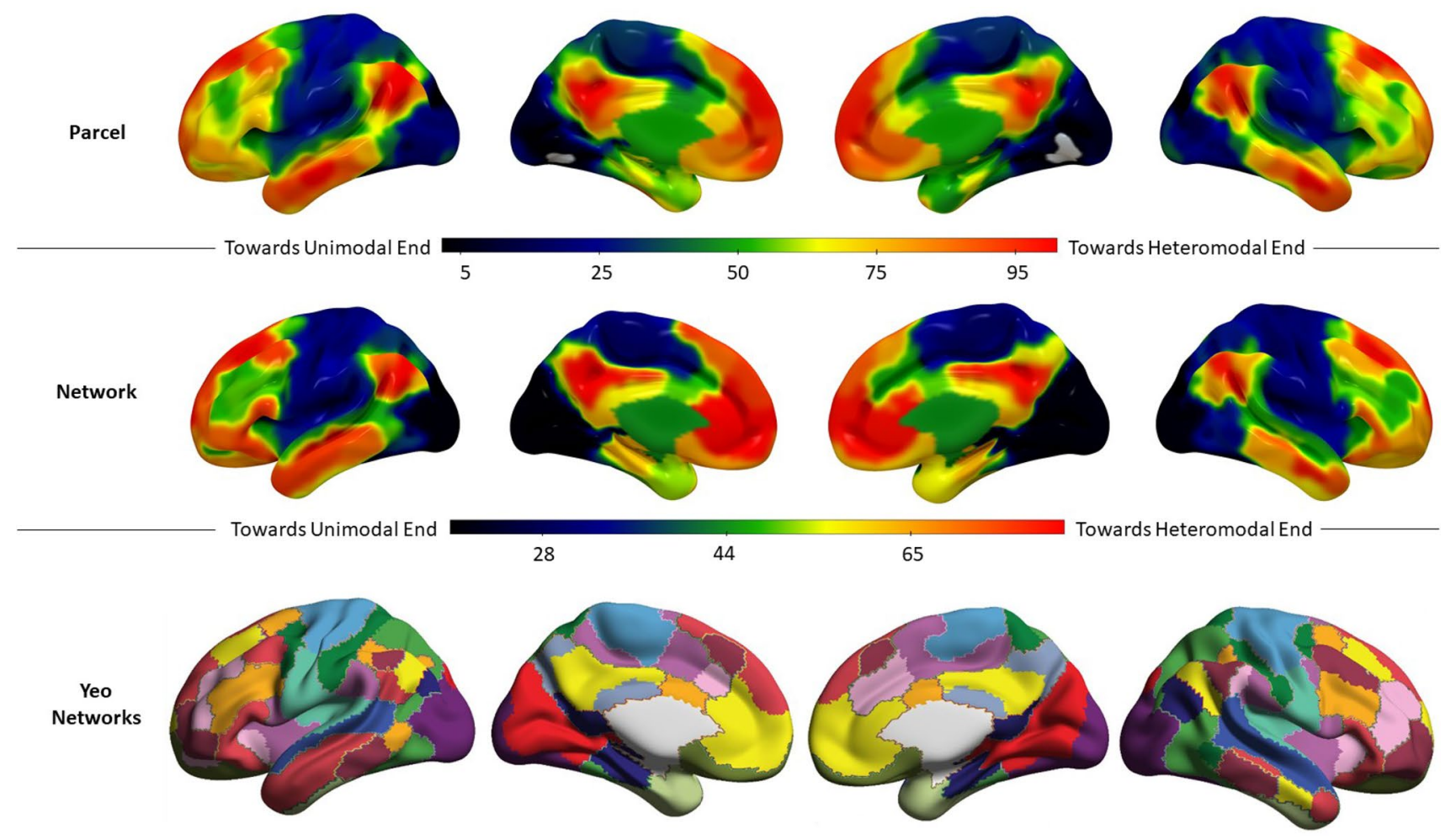

Fig. 3 Top row: Group mean principal gradient value for each parcel in Schaeffer's 400-parcel solution for our sample of 253 participants. Middle row: Group mean principal gradient value for each network in Yeo's 17-network solution for our sample of 253 participants. Gradi- ent units are arbitrary and have been normalised on a 0-100 minmax scale. Bottom row: 17 network parcellation by Yeo et al. (2011; the colour code followed in this figure replicates that of Buckner et al. 2011) 
Fig. 4 Hemispheric differences in principal gradient values across the 17 Yeo networks (z-scored). The warm colours represent principal gradient loadings that are nearer the heteromodal apex in LH compared to RH. Cool colours represent principal gradient loadings that are nearer the heteromodal apex in $\mathrm{RH}$ compared to $\mathrm{LH}$

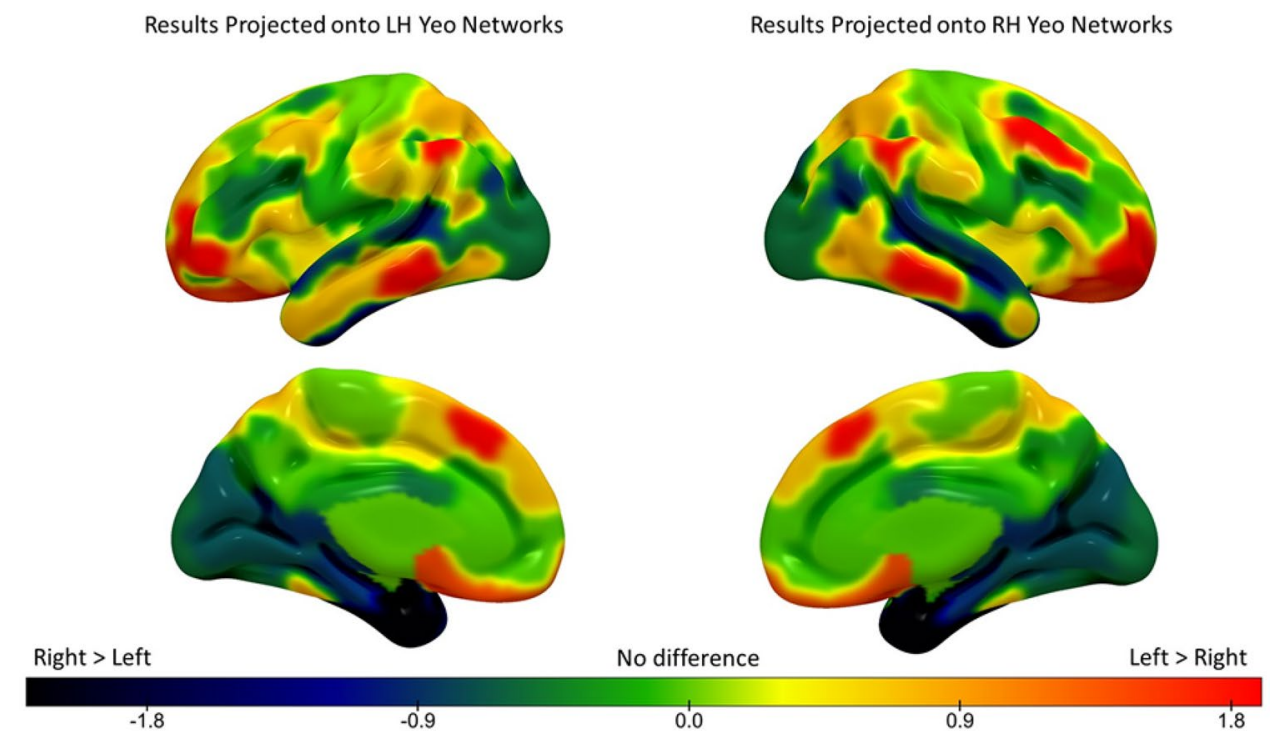

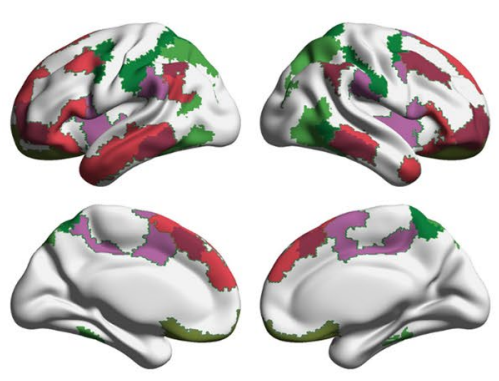

LH closer to heteromodal end
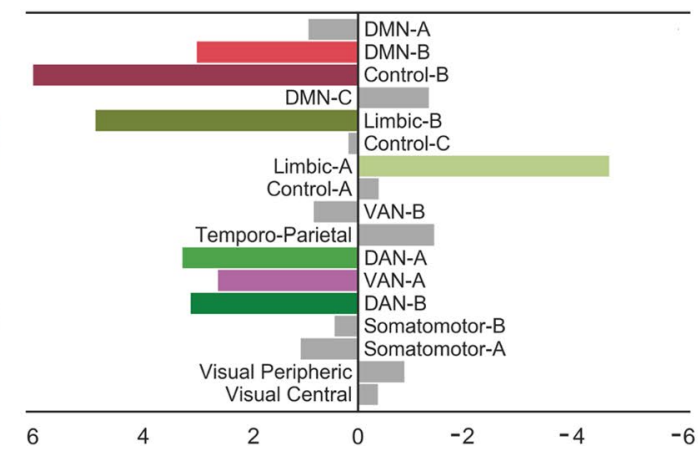
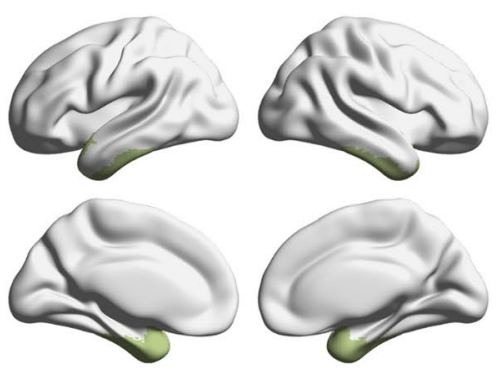

$\mathrm{RH}$ closer to heteromodal end
Fig. 5 Results of permutation testing of $\mathrm{LH}$ versus $\mathrm{RH}$ positions on the principal gradient for each network (5000 simulations, Bonferroni-corrected alpha for 17 comparisons). The size of each bar reflects the normalized empirically observed mean difference across the hemispheres for each network. Coloured bars denote networks that showed significant differences (see Table 1 for exact $p$ values)

\section{Hemispheric difference analysis at the network level}

In order to compare the principal gradient loadings of the regions captured in the 400-region parcellation across the cerebral hemispheres, we averaged all parcels that fell within each network in the left and right hemispheres separately, and then performed a subtraction (left-right) and $z$-scored the resulting differences. The results can be seen in Fig. 4. The principal gradient loadings in warm colours are nearer the heteromodal apex in the left hemisphere compared to the right, and the cool colours represent principal gradient loadings that are nearer the heteromodal apex in the right hemisphere compared to the left. Since Yeo et al. (2011) networks are not symmetrical, subtracting left-right in one network will yield the same value for both hemispheres, but and are colour-coded to indicate the position of each network in the brain. The brain map on the left side of the plot shows networks that were closer to the heteromodal end of the gradient in LH, while the brain map on the right side of the plot shows one network that was closer to the heteromodal end of the gradient in $\mathrm{RH}$

in different topological locations. To visualise this, in Fig. 4 we projected the results of the subtraction for each network (which are the same) onto the left and right hemisphere network maps (which are not the same). The value of these left-right network gradient differences was highly correlated with the principal gradient at the group level $(r=0.93$, $p<0.0001)$, consistent with the expectation that heteromodal cortex shows more divergent connectivity across the hemispheres than unimodal cortex.

We performed a repeated-measures ANOVA to formally test for differences in principal gradient loadings at the network level ( 2 hemispheres by 17 networks), controlling for global hemispheric differences in gradient values by entering each participant's global left-right difference value as a covariate of no interest. The results of this 
Table 1 Normalised (on a scale of 0-100) means across hemispheres for each Yeo network

\begin{tabular}{lllc}
\hline Network & LH mean (SD) & RH mean (SD) & Corrected $p$ value \\
\hline DMN-A & $82.76(5.84)$ & $81.85(5.5)$ & $>0.1$ \\
DMN-B & $77.62(6.2)$ & $74.69(6.88)$ & $.000^{* * *}$ \\
Control-B & $73.29(7.51)$ & $67.39(7.44)$ & $.000^{* * *}$ \\
DMN-C & $64.75(9.98)$ & $66.05(10.4)$ & $>0.1$ \\
Limbic-B & $67.51(10.38)$ & $62.74(9.54)$ & $.000^{* * *}$ \\
Control-C & $59.6(12.14)$ & $59.41(11.38)$ & $>0.1$ \\
Limbic-A & $54.66(9.51)$ & $59.22(8.53)$ & $.000^{* * *}$ \\
Control-A & $52.37(7.54)$ & $52.76(7.59)$ & $>0.1$ \\
Salience/VAN- & $50.67(9.18)$ & $49.85(9.18)$ & $>0.1$ \\
$\quad$ B & & & \\
Temporo- & $48.74(11.07)$ & $50.14(10.37)$ & $>0.1$ \\
$\quad$ parietal & & & $.000^{* * *}$ \\
DAN-A & $39.03(8.61)$ & $35.84(8.35)$ & $.000^{* * *}$ \\
Salience/VAN- & $35.94(7.37)$ & $33.38(7.29)$ & $.000^{* * *}$ \\
$\quad$ A & & & $>0.1$ \\
DAN-B & $34.11(7.57)$ & $31.07(7.3)$ & $>0.1$ \\
Somatomotor-B & $29.47(7.33)$ & $29.03(7.38)$ & $>0.1$ \\
Somatomotor-A & $29.56(7.29)$ & $28.51(7.47)$ & $19.69(8.73)$ \\
Visual periph- & $18.84(8.96)$ & $19.48(7.04)$ & $>0.1$ \\
$\quad$ eric & & & \\
Visual central & $19.11(6.57)$ & 19.46 & \\
\hline
\end{tabular}

Larger values reflect greater proximity to the heteromodal end of the principal gradient. The $p$ values indicate the results of pairwise bootstrapped permutation testing of LH vs RH principal gradient means for each network (5000 simulations)

***=significant at $p<0.0002$ with Bonferroni-correction for 17 comparisons

ANOVA revealed significant main effects of hemisphere $\left[F(1,251)=538.82, p<0.0001, \eta p^{2}=0.68\right]$, and network $\left[F(8.48,2127.52)=902.44, p<0.0001, \eta p^{2}=0.78\right]$, as well as a significant hemisphere by network interaction $[F(10.62$, $2664.95)=18.61, p<0.0001, \eta p^{2}=0.07$; all values with Greenhouse-Geisser correction to account for violations of the sphericity assumption]. Subsequent post-hoc tests comparing the left and right hemispheres for each network (using permutation testing with 5000 simulations to establish significance; Bonferroni-corrected for 17 comparisons) revealed that these hemispheric differences were robust for seven networks: DMN-B, Control-B, Limbic-B, Limbic-A, DAN-A, DAN-B, and VAN-A (Fig. 5). Only these seven networks were carried forward for further analyses. Supplementary Fig. 1 shows the distribution of gradient values for these seven networks.

\section{Behavioural regressions}

We next tested whether the degree of difference in principal gradient loadings across hemispheres for each Yeo network was associated with performance on semantic and non-semantic (visual reasoning and working memory) tasks outside the scanner. We defined regression models using the empirically observed mean hemispheric difference in gradient scores (left-right hemispheres) for each network as the dependent variable, and the efficiency of semantic decisions, maximum number of items remembered in digit span, and accuracy on Raven's matrices as three explanatory variables per participant. The model also included efficiency in a perceptual matching task matched superficially to the semantic tasks. There was a significant association between task performance and hemispheric gradient differences for two out of seven networks (only networks showing a significant difference on the principal gradient in the analysis above were included). Hemispheric differences in gradient values for Control-B showed a positive association with overall semantic performance $(\beta=0.19, p=0.02)$, and no relationship with working memory $(\beta=0.05, p>0.1)$ or visual reasoning $(\beta=0.003, p>0.1)$. DAN-B showed a negative association between left-right hemisphere gradient loadings and visual reasoning $(\beta=-0.16, p=0.02)$ and no relationship with semantic performance $(\beta=-0.1, p>0.1)$ or working memory $(\beta=-0.13, p=0.07)$ (see Fig. 6). In sum, participants whose Control-B network was closer to the heteromodal DMN end of the principal gradient in the left hemisphere compared with the right showed more efficient semantic retrieval; in contrast when the DAN-B network was closer to the heteromodal end of the principal gradient in the right hemisphere compared with the left, participants showed better visual reasoning on a matrices task. There were no significant associations with working memory. An additional analysis comparing subtasks of the semantic battery observed no significant association between gradient hemispheric differences and the effects of the modality of presentation (pictures versus words) or strength of association (weak versus strong associations), with all $p$ values $>0.1$ (see supplementary materials, Table $\mathrm{S} 1$ ); these results are in line with the PCA reported in Sect. "Materials". (Semantic dimensionality reduction), showing that the variance of this task can be explained with one single factor. All these analyses controlled for differences between the semantic and non-semantic tasks by including a perceptual task matched in presentation and response requirements with the semantic task (see Sect. "Materials": 'Perceptual figure matching task'). The efficiency scores of this perceptual task showed a significant negative association with the DAN-B hemispheric difference scores $(\beta=0.19, p=0.02)$, but no association with the Control-B hemispheric difference scores $(\beta=0.02, p>0.1)$.

Given that individual differences in semantic cognition predicted hemispheric differences in principal gradient values, we next asked if Control-B and DMN networks are closer on the principal gradient of connectivity in the left hemisphere compared with the right. This finding would be 

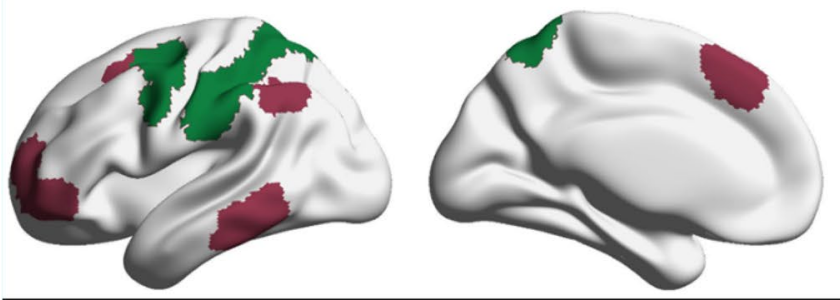

Control B
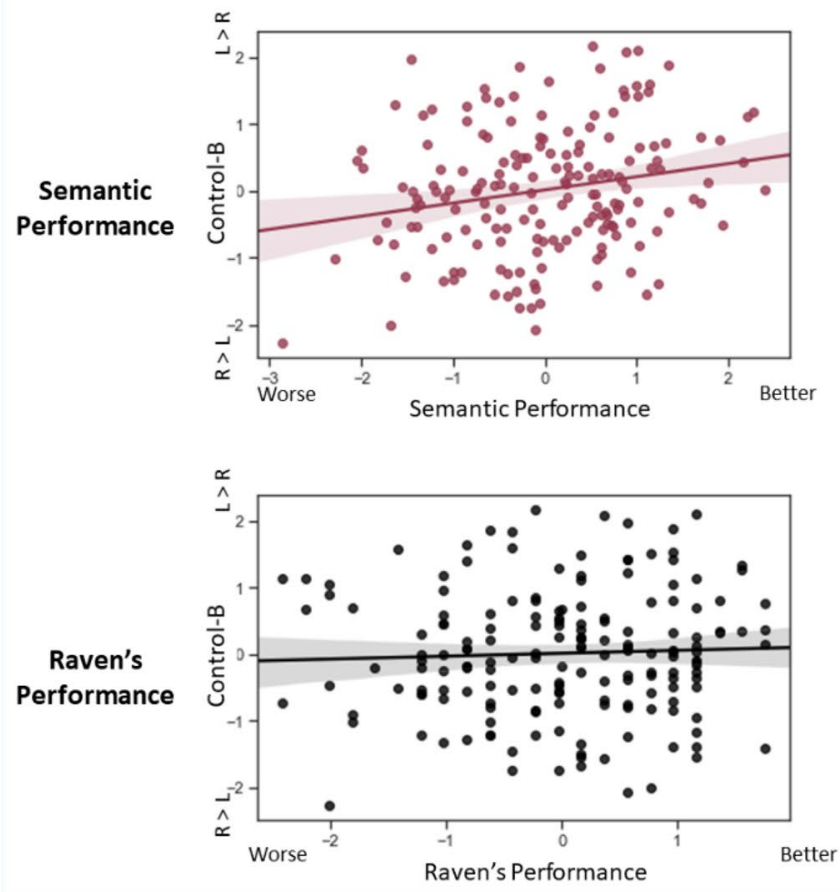

Fig. 6 Scatterplots showing the relationship between hemispheric difference scores on the principal gradient and efficiency on semantic decisions (middle row) and accuracy on a visual reasoning task (Raven's progressive matrices; bottom row). Only networks with sig-

consistent with the greater coupling of these networks in states of controlled semantic retrieval reported by Davey et al. (2016). Yet the sensitivity of the principal gradient to this pattern of functionally-relevant network similarity in the left hemisphere is not yet established since both Control-B and DMN-B (the adjacent network on the principal gradient) showed higher gradient values for the left hemisphere compared with the right in the analysis in Fig. 5.

We computed the distance on the principal gradient between Control-B and the two networks that were closer to the heteromodal apex of the principal gradient (DMN-A and DMN-B) for each hemisphere separately and compared these distances across hemispheres using paired $\mathrm{t}$-tests (all $\mathrm{p}$ values reported are corrected for multiple comparisons). There was a significantly smaller difference in principal gradient values between Control-B and DMN-B in the left hemisphere compared with the right (mean difference in $\mathrm{LH}=4.33, \mathrm{SD}=7.4$; and in $\mathrm{RH}=7.29$,
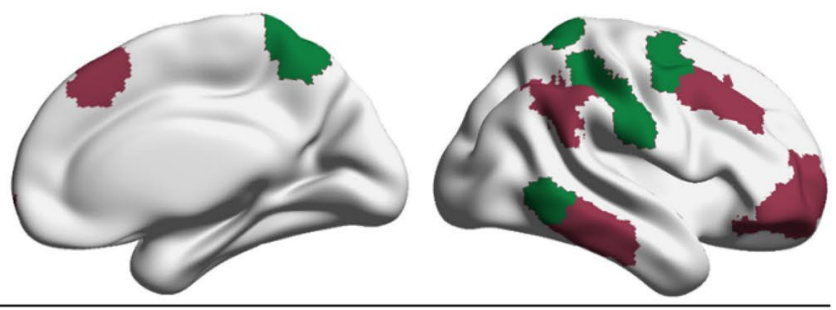

DAN-B
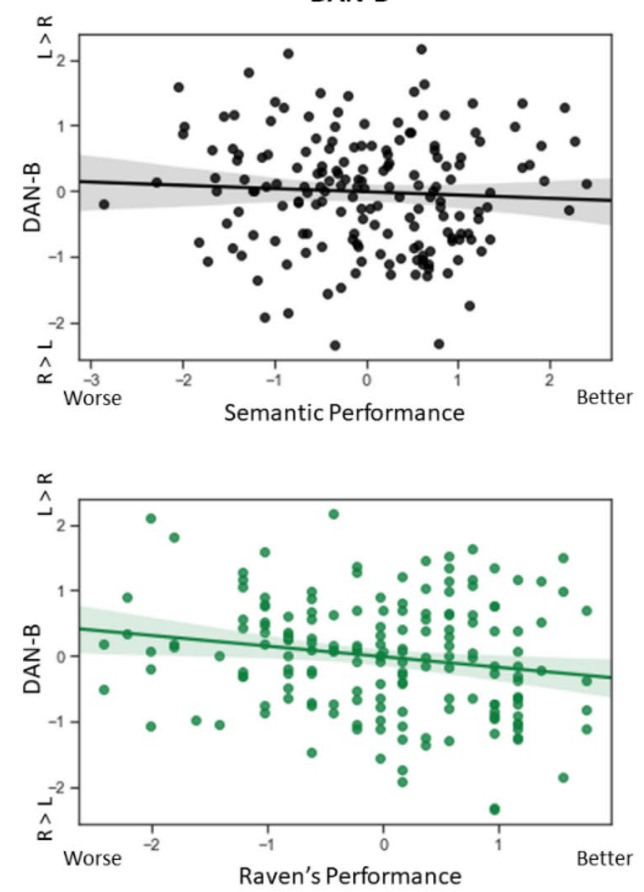

nificant results are shown (Control $\mathrm{B}$ on the left-hand side; DAN-B on the right-hand side). The scatterplots in colour denote significant effects in the regression model and have been colour coded to the networks driving the effect, shown in the top row

$\mathrm{SD}=7.93 ; t(174)=6.52, p<0.001)$. There was a similarly smaller difference in principal gradient values between Control-B and DMN-A in the left hemisphere compared with the right (mean difference in $\mathrm{LH}=9.47, \mathrm{SD}=7.77$; and in $\mathrm{RH}=14.45, \mathrm{SD}=7.06 ; t(174)=13.07, p<0.001$ ). This confirms that Control-B is closer to DMN along the principal gradient.

We repeated this analysis to establish if DAN-B has greater proximity to sensorimotor networks in the right hemisphere compared with the left. There were significantly smaller gradient distances in the right hemisphere compared with the left for all four relevant network comparisons: (i) visual central: $\mathrm{LH}=15.01, \mathrm{SD}=8.7 ; \mathrm{RH}=11.6$, $\mathrm{SD}=9.09 ; t(174)=7.96, p<0.001)$; (ii) visual peripheral: $\mathrm{LH}=15.28, \mathrm{SD}=11.33 ; \mathrm{RH}=11.37, \mathrm{SD}=11.47$; $t(174)=8.76, p<0.001)$, (iii) somatomotor-A $(\mathrm{LH}=4.55$, $\mathrm{SD}=5.93 ; \mathrm{RH}=2.55, \mathrm{SD}=5.26 ; t(174)=7.95, p<0.001)$ and (iv) somatomotor- $\mathrm{B}(\mathrm{LH}=4.64, \mathrm{SD}=5.12 ; \mathrm{RH}=2.04$, 


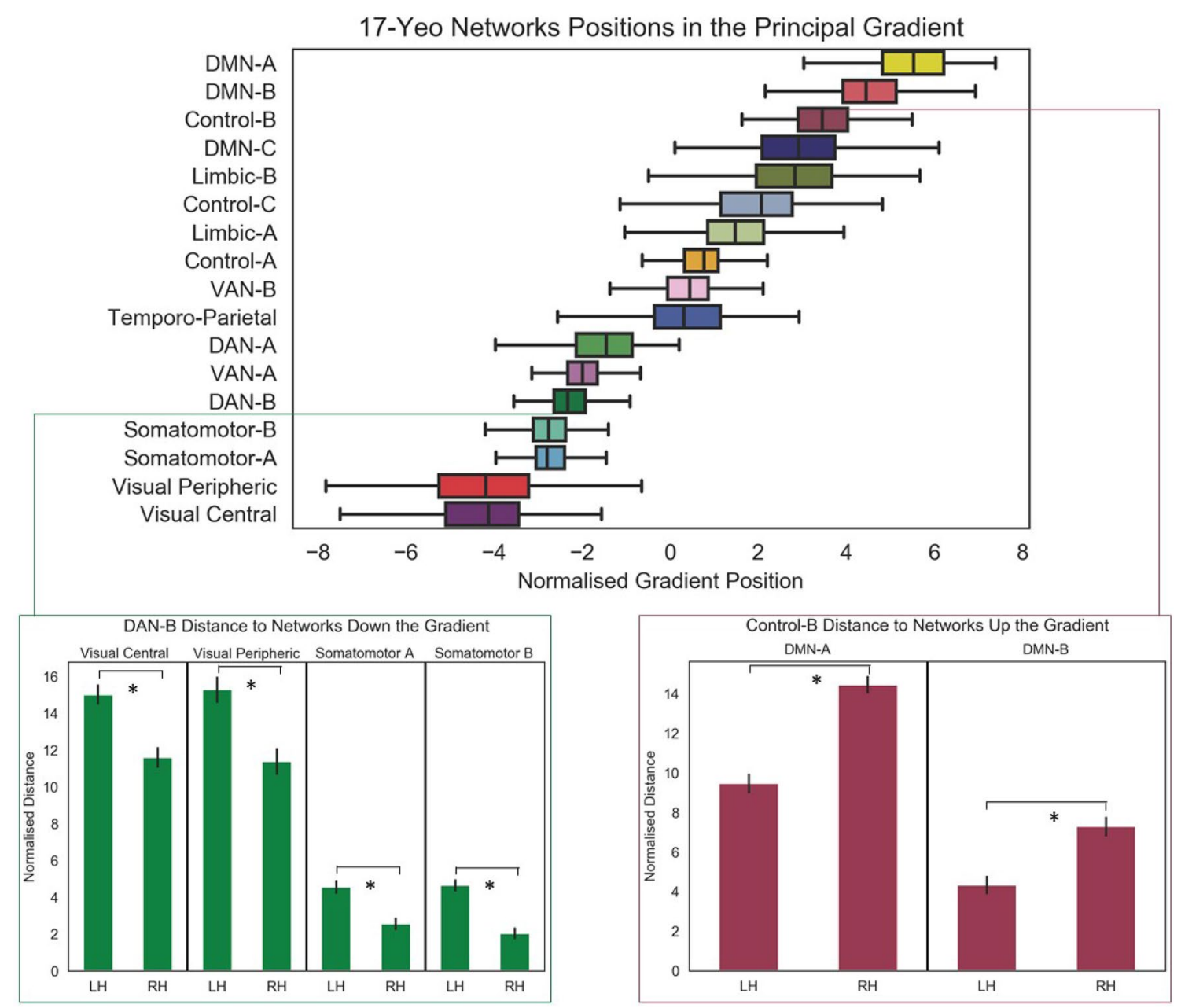

Fig. 7 Differences of network positions in the principal gradient between pairs of networks that are relevant for lateralised cognitive processes. $*=p<.001$ (corrected for multiple comparisons)

$\mathrm{SD}=4.88 ; t(174)=9.75, p<0.001)$. DAN-B was closer to all sensorimotor networks in the right hemisphere compared with the left. These results can be seen in Fig. 7.

\section{Study 2}

\section{Behavioural results}

As reported in Gao et al. (2021), equal numbers of word pairs were judged to be related or unrelated by the participants in the semantic task [mean ratio 0.491 vs. 0.495 , $\left.\chi^{2}(1)=0.00021, p>0.995\right]$. Linear mixed-effects models examined whether associative strength and WM load were reliable predictors of behaviour. Gao et al. showed that both the strength of the semantic association (word2vec value) and WM load successfully manipulated task difficulty. For the semantic task, the continuous word 2 vec value was positively associated with a higher probability that participants would identify a semantic relationship between the words $\left[\chi^{2}(1)=2421.3, p<0.001\right]$ in a logistic regression. When word pairs were grouped into 5 levels according to their word2 vec value, the relationship was still significant $\left[\chi^{2}(1)=2467.8, p<0.001\right]$.

Regarding the validity of the novel manipulation of semantic control used in this task, linear mixed effects models showed that association strength modulated RT in a manner consistent with the deployment of semantic control: trials in the 'related' category with higher word2vec scores should involve less effort, since there is a readily available context, whereas those with lower word2vec scores require control for establishing a context; the reverse pattern is expected for trials judged to be 'unrelated', where a high word2vec score means the context must be effortfully suppressed, while a low word$2 \mathrm{vec}$ score makes the link easier to reject, since no shared context exists. Linear mixed-effects analyses, including participant as a between-subject variable and association level as a within-subject variable, showed associative 
strength was negatively associated with $\mathrm{RT}$ for related trials $\left[\chi^{2}(1)=146.6, p<0.001\right]$, and positively associated with RT for unrelated trials $\left[\chi^{2}(1)=58.668, p<0.001\right]$. It was more difficult for participants to retrieve a semantic connection between two words when the strength of association was lower; on the contrary, it was easier for them to decide there was no semantic connection between word pairs with low word2vec values.

For the WM task, the proportion of correct responses was $84.8 \%$, when all memory load levels were considered. The more items to be maintained or manipulated in WM, the more difficult the trial was expected to become. A logistic regression showed that higher WM load was associated with lower accuracy $\left[\chi^{2}(1)=112.4, p<0.001\right]$. A further linear mixed-effects model with participant as a between-subject variable and memory load as a withinsubject variable revealed a significant positive relationship between load level and RT for correct responses $\left[\chi^{2}(1)=39.826, p<0.001\right]$.

A two-way repeated-measures ANOVA examining the effects of task condition ('related', 'unrelated' and 'WM correct') and difficulty level (5 levels) on the proportional change in RT for each difficulty level of the task, relative to the average RT for each condition, showed a significant interaction between task condition and difficulty level $[F(5.40,134.88)=8.33, p<0.001$, Greenhouse-Geisser corrected] along with a main effect of difficulty level $[F(3.13,78.35)=53.26, p<0.001$, Greenhouse-Geisser corrected]. Together, these results suggest that association strength and WM load successfully manipulated task difficulty, engaging effortful cognition. Summary measures of the behavioural data reported here are provided in Supplementary Table 6 and Supplementary Fig. 10.

\section{Parametric effects of control demands}

Study 1 found that when Control-B is closer to the DMNend of the principal gradient in the left hemisphere versus the right, participants have more efficient semantic retrieval. In contrast, when DAN-B is closer to the heteromodal end of the principal gradient in the right hemisphere, participants show better visual reasoning on a progressive matrices task. These findings predict a hemispheric dissociation between networks in the effects of control demands across domains (i.e., in effects of semantic control demands and non-semantic difficulty-even within the verbal domain). In Study 2, we tested this prediction by examining the effects of parametric manipulations of semantic control demands (strength of association) and verbal working memory load on activation within the left and right hemisphere components of control-B and DAN-B networks (this data set did not include a visual reasoning task). An omnibus ANOVA examining the factors of hemisphere (left vs. right), task difficulty (related semantic, unrelated semantic and working memory) and network (Control-B vs. DAN-B) showed a three-way interaction between these factors $[F(2,54)=6.71$, $p=0.003]$.

Separate repeated-measures ANOVAs for control-B and DAN-B found distinct patterns. Control-B showed a significant interaction between hemisphere and condition, reflecting larger effects of control demands in the semantic task relative to working memory $[F(1.34,36.07)=7.72, p=0.005$, $\left.\eta p^{2}=0.22\right]$. Post-hoc tests revealed a greater response to difficulty for semantic decisions in the left hemisphere versus the right $(p<0.001)$; in contrast, there were no hemispheric differences in the effect of WM load. There was no task by hemisphere interaction for DAN-B $(F<1)$. There were also no main effects of task in either network, but there was
Table 2 Results of the ANOVAs on the parametric difficulty effect maps

\begin{tabular}{|c|c|c|c|c|c|}
\hline Model & Effect & $d f$ & $F$ & $p$ & $\eta p^{2}$ \\
\hline \multirow[t]{7}{*}{ Omnibus } & Interaction $^{+}$ & $1.55,41.85$ & 6.71 & 0.006 & 0.199 \\
\hline & Task*hemisphere $^{+}$ & $1.35,36.4$ & 5.07 & 0.021 & 0.158 \\
\hline & Task*network & 2,54 & 1.42 & 0.25 & 0.05 \\
\hline & Hemisphere*network & 1,27 & 6.81 & 0.015 & 0.201 \\
\hline & Hemisphere & 1,27 & 37.9 & $<0.0001$ & 0.584 \\
\hline & Network & 1,27 & 9.59 & 0.005 & 0.262 \\
\hline & Task & 2,54 & 0.26 & 0.77 & 0.89 \\
\hline \multirow[t]{3}{*}{ Control-B } & Interaction $^{+}$ & $1.33,36.07$ & 7.72 & 0.005 & 0.222 \\
\hline & Hemisphere & 1,27 & 27.23 & $<0.0001$ & 0.502 \\
\hline & Task & 2,54 & 0.16 & 0.851 & 0.006 \\
\hline \multirow[t]{3}{*}{ DAN-B } & Interaction $^{+}$ & $1.62,43.62$ & 0.21 & 0.768 & 0.008 \\
\hline & Hemisphere & 1,27 & 18.49 & $<0.0001$ & 0.406 \\
\hline & Task & 2,54 & 2.25 & 0.116 & 0.077 \\
\hline
\end{tabular}

The effects marked with + were subjected to Greenhouse-Geisser corrected since our data violated the assumption of sphericity (Mauchley's test of sphericity $p<.05$ in both cases) 
more activation in the left hemisphere overall, likely reflecting the verbal nature of the tasks. Full ANOVA results are reported in Table 2 . These results confirm that the left-lateralised components of Control-B show a specific response to semantic control demands, but not to working memory load. In contrast, DAN-B shows no difference in response to these two forms of verbal control across hemispheres. These effects are shown in Fig. 8.

\section{Discussion}

This study investigates the lateralisation of function along the principal gradient-a key topographical component of large-scale intrinsic connectivity that captures the separation of unimodal and heteromodal cortex (Margulies et al. 2016). We show that intrinsic connectivity patterns in the two hemispheres are situated at different points along the principal gradient: overall, left hemisphere parcels are closer to the heteromodal end of the principal gradient than right hemisphere parcels, consistent with the role of this hemisphere in key heteromodal functions, such as semantic cognition and language. This pattern was observed in many canonical heteromodal networks derived from a whole-brain parcellation of resting-state data (Yeo et al. 2011), including control, default, dorsal and ventral attention networks; however, this pattern was inverted for Limbic-A, centred on the ventral anterior temporal lobe (ATL). There was also no gradient difference between the hemispheres in sensorimotor networks. In Study 1, individual differences in the relative gradient positions of networks across the hemispheres were found to have functional associations with two cognitive processes with opposing patterns of lateralisation, semantic cognition and visual reasoning (there were no effects for working memory). Participants whose Control-B network was closer to the heteromodal DMN end of the principal gradient in the left hemisphere compared with the right showed more efficient semantic retrieval; in contrast when the DAN-B network was closer to the heteromodal end of the principal gradient in the right hemisphere compared with the left, participants showed better visual reasoning on a progressive matrices task. Finally, in Study 2, we established that Control-B dissociates from DAN-B in the effect of verbal task demands on task activation in the left and right hemispheres. Control-B shows a left-lateralised
Fig. 8 Parametric effects of difficulty in semantic and non-semantic tasks for 26 participants in Control B and DAN-B Yeo networks, split by hemisphere (the error bars depict the standard error of the mean), with effects expressed in percentage signal change
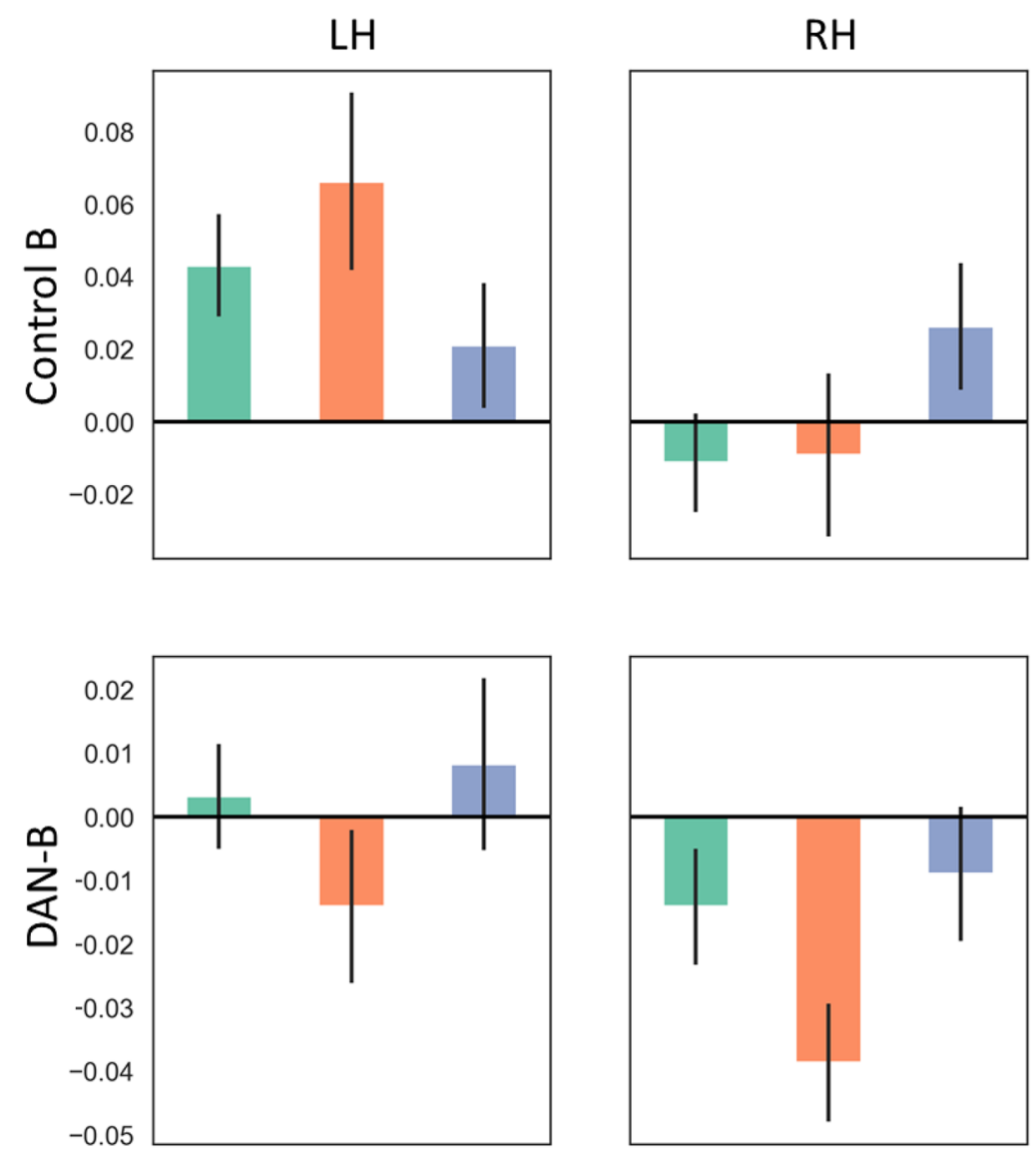

Semantic Related
Working Memory 
response to semantic control demands but not working memory load, consistent with the view that lateralised control regions near the DMN apex of the principal gradient support controlled semantic retrieval.

To date, only one previous study has attempted to describe hemispheric differences in the principal gradient (Liang et al. 2021). Despite important differences in methodology, our findings align with Liang et al.'s study: both investigations found higher gradient values in the left than right hemisphere for ventromedial prefrontal cortex, IFG and lateral ATL. However, Liang et al. extracted separate gradients for the left and right hemispheres and considered these patterns within a 7-network parcellation; consequently, they could not identify the sub-network hemispheric differences that we observed, or directly compare left and right hemisphere networks within the same decomposition. The study by Liang et al. also did not assess the functional significance of hemispheric differences on the principal gradient, which was the main focus of the current study.

We found that left hemisphere parcels were, in general, closer than right hemisphere parcels to the DMN apex of the principal gradient, helping to explain why key heteromodal functions-such as language and semantic cognition-are left-lateralised. Margulies et al. (2016) found that the terms LANGUAGE:SYNTAX and LANGUAGE:SEMANTICS were among the BrainMap behaviour terms closest to the heteromodal end of the principal gradient; similarly, VERBAL SEMANTICS was towards the heteromodal apex in Neurosynth (a meta-analytic tool; Yarkoni et al. 2011). Language and semantics both depend on the retrieval of heteromodal representations-extracted from diverse sensory-motor features when we acquire concepts and words; moreover, they both require retrieval to be controlled to fit rapidly changing goals and contexts. These different components of semantic cognition-conceptual representations plus control processes - are lateralised to different degrees (Gonzalez Alam et al. 2019). Semantic control processes are supported by a strongly left-lateralised network, encompassing left inferior frontal gyrus, and left posterior middle and inferior temporal cortex (Jackson 2021; Noonan et al. 2013). The resting-state functional connectivity between these semantic control sites is stronger in the left hemisphere compared with the right (Gonzalez Alam et al. 2019). In contrast, heteromodal conceptual representation is thought to be supported by bilateral ventral ATL (Ding et al. 2020; Lambon Ralph et al. 2017; Patterson et al. 2007). Evidence for bilateral conceptual representation in ventral ATL is provided by neuroimaging studies (Bright et al. 2004; Tranel et al. 2005; Vandenberghe et al. 1996; Visser et al. 2009, 2011) and neuropsychology; patients with bilateral ventrolateral ATL damage show severe semantic impairment (for example, in semantic dementia), while patients with unilateral lesions have milder deficits (Rice et al. 2018).
This difference between strongly lateralised semantic control processes and bilateral conceptual representations may help to explain why Limbic-A, centred on the ventral anterior temporal lobe, was situated closer to the DMN end of the principal gradient in the right hemisphere compared with the left. Gonzalez Alam et al. (2019) found that right ATL was more connected to core DMN regions, including angular gyrus and dorsomedial prefrontal cortex; in contrast, left ATL was more connected to left-lateralised sites implicated in semantic control, including left intraparietal sulcus and left anterior insula bordering ventral parts of inferior frontal gyrus. In the left hemisphere, the principal gradient captures the order of networks from DMN, through the semantic control network, to executive regions (Wang et al. 2020). As a consequence, this proximity (and shared connectivity) of left ATL to semantic control regions might explain the unique gradient difference in Limbic-A. Right hemisphere components of this network might be closer to the heteromodal apex of the principle gradient because they are further from left-lateralised control networks situated towards the middle of the gradient.

The left-lateralised semantic control network is thought to be partially distinct from multiple demand cortex that responds to executive demands across domains: for example, effects of semantic but not non-semantic control demands are observed in anterior aspects of inferior frontal gyrus and posterior middle temporal gyrus (Davey et al. 2015, 2016; Hoffman et al. 2010; Jackson 2021; Noonan et al. 2013; Whitney et al. 2011, 2012). Similarly, the frontoparietal control network, defined through analyses of intrinsic functional connectivity, shows a bipartite organisation (Dixon et al. 2018), overlapping with Control-A and Control-B networks within the Yeo et al. (2011) parcellation used in this study. Dixon et al.'s (2018) control subnetwork including more anterior parts of both inferior prefrontal cortex and middle temporal gyrus has a topographical distribution that is similar to the functionally defined semantic control network (Jackson 2021; Noonan et al. 2013), and shows stronger interactions with DMN regions than the other control subnetwork. Similarly, the functionally defined semantic control network shows relatively strong intrinsic connectivity to both DMN, associated with heteromodal integration or abstraction, and domain-general executive and attention networks (Davey et al. 2016). This pattern of connectivity may allow states of controlled semantic cognition in which ongoing activation within DMN regions is shaped through the application of goal representations within the executive cortex to promote more weakly encoded aspects of knowledge (Wang et al. 2020). This finding is consistent with our observation of more efficient semantic cognition when the Control-B network was closer on the principal gradient to DMN in the left hemisphere as opposed to the right. Gradient differences between the two hemispheres might allow 
one control subnetwork to connect more strongly with DMN, supporting semantic control in the left hemisphere, while the other control subnetwork in the right hemisphere connects more strongly with sensory-motor regions, with advantages for demanding tasks that are oriented towards external sensory-motor features. This possibility is consistent with Wang et al. (2014) who found that control network regions in the left hemisphere have stronger connectivity with DMN, while right hemisphere control sites are closer in connectivity to attentional networks.

Like the frontoparietal regions linked to cognitive control, DMN also has subnetworks; this study provides some evidence that these subdivisions within control and DMN networks are functionally related. Just as we found a control network that was closer to the heteromodal end of the principal gradient in the left hemisphere, DMN-B (the adjacent network), showed the same pattern. DMN-B includes regions such as lateral ATL, angular gyrus, inferior frontal gyrus and dorsomedial prefrontal cortex that are associated with semantic processing in the left hemisphere (Jackson 2021; Jefferies 2013; Lambon Ralph et al. 2017; Noonan et al. 2013; Rice et al. 2015b), and this DMN variant has repeatedly shown functional dissociations with core DMN regions such as posterior cingulate cortex and more ventromedial prefrontal regions (Chiou et al. 2020; Zhang et al. 2020), referred to here as DMN-A. DMN-B is associated with lateralised cognitive processes, like language and semantics, as well as social cognition (Andrews-Hanna et al. 2014). This network shows responses to externally generated, conceptual tasks, including those that interface with perception. In contrast, DMN-A or core DMN is thought to be more detached from perception, and is engaged by internally generated, self-referential and autobiographical memory processing (Chiou et al. 2020). It is interesting to note that it is DMN-B, not core DMN, that shows a lateralised position on the principal gradient. This is consistent with the possibility that lateralisation reflects the need to sustain and/or control heteromodal semantic retrieval (as opposed to the need to support internally-generated mental states, which are also associated with the heteromodal end of the principal gradient).

We found evidence of significant differences in lateralisation patterns within attentional networks as well, with both DAN and VAN falling closer to the heteromodal end of the gradient in the left hemisphere. Although attention has been traditionally conceptualised as a right-lateralised cognitive function, contemporary neuroscientific research paints a more nuanced picture with complex patterns of lateralisation across the traditionally accepted ventral and dorsal attention networks (Corbetta and Shulman 2002; Jeong and Xu 2016; Szczepanski et al. 2010; Thiebaut de Schotten et al. 2011a, b). Critically, the DAN also plays a role in the flexible coupling of the control network across hemispheres and subdivisions (Dixon et al. 2018; Wang et al. 2014). Both DAN and control networks showed significant but opposing behavioural associations in our individual differences' analysis of the position of networks on the principal gradient across hemispheres. Hemispheric differences in DAN-B were related to Raven's matrices performance, but in contrast to semantic cognition, participants whose DAN-B was closer to the heteromodal end of the gradient in the right hemisphere were better at the task. Performance in reasoning tasks relies on efficient interregional communication within the bilateral multiple-demand system, and between this control network and other regions, for example, areas that maintain visuo-spatial representations, to orchestrate complex cognition (Gläscher et al. 2010; Shin and Jeon 2021). Moreover, previous research has linked performance on progressive matrices to attentional capacity (Schweizer and Moosbrugger 2004), and performance can also be decomposed into in two components relating to perceptual and executive attention (Ren et al. 2012), with the latter corresponding more closely to the DAN (Corbetta et al. 2008; Corbetta and Shulman 2002) and accounting for more variance in visual reasoning tasks (Ren et al. 2013). The right hemisphere is particularly activated during the performance of this task in certain conditions (Bishop et al. 2008; Prabhakaran et al. 1997). Contrasting specific types of reasoning tasks, like matrix and analogical reasoning, reveals greater right-lateralised responses in fronto-parietal regions for matrix reasoning (Hobeika et al. 2016). Right frontal regions in this network also show a greater response as matrix tasks increase in complexity (Krawczyk et al. 2010a, b, 2011). Consequently, higher DAN gradient values in the right hemisphere might reflect closer integration of DAN and control networks in the right hemisphere, which facilitates the efficient deployment of attention to solve spatial relational reasoning problems.

We also compared semantic cognition with verbal WM tasks and found no lateralisation effects within these networks for the latter. This suggests that the lateralisation of semantic cognition is related to the controlled retrieval of conceptual representations as opposed to the need to use language in the task, and is compatible with accounts of WM demands engaging bilateral multiple demand cortex (Duncan, 2001, 2010; Fedorenko et al. 2013; Hugdahl et al. 2015). However, we do not provide a full description of lateralisation in verbal working memory, since Study 1 did not find any associations between digit span and hemispheric gradient differences for any networks; consequently networks relevant to working memory were not selected for analysis in Study 2.

There are several limitations of the current study. The tasks compared in Study 1 (semantic battery versus digit span and Raven's progressive matrices) varied in multiple ways, including mode of response, task instructions, time 
allowed to respond and level of demand. In the analysis examining the association between individual differences in gradient lateralisation and semantic performance, we statistically controlled for performance on a visual matching task with similar input and response characteristics to the semantic task, helping to ensure that semantic task effects reflected the requirement for conceptual retrieval. The fact that we observed an association with hemispheric differences in gradient position in DAN-B but not in Control-B with this perceptual task, while the association of hemispheric differences in Control-B with semantic performance remained significant, makes it unlikely that differences between the semantic and non-semantic tasks gave rise to our results. However, there were no control tasks for digit span or visual reasoning; moreover, hemispheric differences in the principal gradient might be related to other non-semantic cognitive domains not assessed here. Furthermore, the parametric manipulations of difficulty in WM and semantic judgements in Study 2 are not analogous since we manipulated working memory load (i.e., items to be maintained) and the strength of the semantic association (i.e., semantic distance as measured by word2vec). As noted by Gao et al. (2021), the WM task was associated with faster responses than the semantic control task, perhaps because word reading takes longer than letter identification, but reading times are not necessarily relevant to the activation of control networks. Another difference among these tasks was that strength of association had a larger effect on RT than working memory load, although RT does not provide a direct measure of cognitive control demands. We selected these manipulations because the literature shows that they robustly vary the activation of control regions (Noonan et al. 2013; Jackson 2021; Fedorenko et al. 2013; Emch et al. 2019); however, it would also be possible to manipulate control demands in a more comparable way across these domains, for example, by varying the strength of the distractors in both tasks.

Our methods also did not allow us to investigate the source of the network asymmetries at the sub-network or parcel level, since the parcellation we used (Schaefer et al. 2018) did not provide homotopic regions that could be compared (see Popovych et al. 2021, for the effect of parcellation choice on resting-state results). The parcels we used from Schaefer et al. (2018) are derived separately for each hemisphere, and there are an unequal number assigned to each network across hemispheres. For example, the DAN-B network has 13 parcels in the left hemisphere organised in three subdivisions (postcentral, frontal eye fields and precentral ventral region), while it has only 11 in the right hemisphere (lacking the precentral ventral region and sporting only 8 postcentral parcels, opposed to 9 in the left hemisphere). These differences might give rise to local restingstate functional connectivity gradients that are present in one hemisphere but not the other (Gordon et al. 2016), and these differences in local organisation could have functional significance. Future research could therefore seek to verify these patterns using symmetrised parcellations (Glasser et al. 2016; Joliot et al. 2015), or use methods that exploit voxel-level timeseries homotopy (Gotts et al. 2013; Jo et al. 2012).

Another limitation is shared by many studies that employ dimensionality reduction methods: the number of dimensions retained for analysis is somewhat arbitrary (Supplementary Figure S6 shows that there is no clear plateauing of the eigenvalues). Here we focussed on the asymmetry of the principal gradient, as it captures the most variance and is known to be important for cognition as well as the organisation of large-scale networks on the cortical surface (Murphy et al. 2018, 2019; Turnbull et al. 2020; Wang et al. 2020). We also provide a supplemental analysis of gradient asymmetries in Gradient 2, but we opted not to extend the analysis to gradients explaining less variance as their interpretability is expected to be lower; moreover, studies have shown functional associations with Gradients 1 and 2, but not with Gradient 3 and beyond (Hong et al. 2019; Murphy et al. 2018, 2019; Turnbull et al. 2020; Wang et al. 2020). Future research could take a different approach by extracting a very large number of gradients, and then identify lowerorder gradients that specifically capture hemispheric differences in higher-order gradients (see Valk et al. 2020).

Finally, it remains unclear why attentional networks (DAN-A; DAN-B and VAN-A) were closer to the heteromodal end of the principal gradient in the left hemisphere, even when the opposite pattern for DAN-B (closer proximity to heteromodal cortex in the right hemisphere) was associated with better visual attention. One possibility is that these attention networks can also support controlled semantic cognition, to varying degrees across people, and that these patterns of left-lateralised and right-lateralised connectivity are in competition. Future research could test whether the position of networks along the principal gradient relates to their capacity for efficient interaction, and whether there are differences in physical distance along the cortical surface in the two hemispheres that reflect the connectivity gradient differences we described.

\section{Conclusions}

We found that networks associated with higher-order cognition in the left hemisphere are positioned closer to the heteromodal end of the principal gradient, including the DMN, control, limbic and attentional networks; in contrast, there were no differences in sensorimotor networks, in line with the literature on functional homotopy. The controlDAN dissociation we observed is compatible with recent proposals of a "inward-outward" organisational principle 
for control networks that differs across the hemispheres, with a privileged interaction of DMN-B and Control-B in the left hemisphere (Dixon et al. 2018; Wang et al. 2014). Individual difference analysis showed that relative network position across the hemispheres is associated with the efficiency of lateralised cognitive processes: proximity of DMN to control regions in the left hemisphere was associated with better semantic processing, while the proximity of DAN to control regions in the right hemisphere was associated with better visual reasoning. Analysis of task-based fMRI data in a separate study showed differential recruitment of the Control-B network across the hemispheres in response to semantic demands but not working memory load.

Supplementary Information The online version contains supplementary material available at https://doi.org/10.1007/s00429-021-02374-w.

Funding The research was supported by European Research Council [FLEXSEM-771863].

Availability of data and material Gradient maps one to ten from the group-averaged dimension reduction analysis described in Sect. Neuroimaging below are publicly available on NeuroVault in a collection (https://neurovault.org/collections/6746/). Raw fMRI and questionnaire data are restricted in accordance with ERC and EU regulations.

Code availability All code used in the production of this manuscript is available from the corresponding authors upon reasonable request.

\section{Declarations}

Conflicts of interest The authors have no conflicts of interest to declare that are relevant to the content of this article.

Ethics approval The study was approved by the York Neuroimaging Centre Ethics Committee. This study was performed in line with the principles of the Declaration of Helsinki.

Consent to participate Written informed consent was obtained for all participants.

Consent for publication Consent for publication of anonymised, grouplevel data was provided as part of the written informed consent.

Open Access This article is licensed under a Creative Commons Attribution 4.0 International License, which permits use, sharing, adaptation, distribution and reproduction in any medium or format, as long as you give appropriate credit to the original author(s) and the source, provide a link to the Creative Commons licence, and indicate if changes were made. The images or other third party material in this article are included in the article's Creative Commons licence, unless indicated otherwise in a credit line to the material. If material is not included in the article's Creative Commons licence and your intended use is not permitted by statutory regulation or exceeds the permitted use, you will need to obtain permission directly from the copyright holder. To view a copy of this licence, visit http://creativecommons.org/licenses/by/4.0/.

\section{References}

Andrews-Hanna JR, Smallwood J, Spreng RN (2014) The default network and self-generated thought: component processes, dynamic control, and clinical relevance. Ann NY Acad Sci 1316:29-52. https://doi.org/10.1111/nyas. 12360

Badre D (2008) Cognitive control, hierarchy, and the rostro-caudal organization of the frontal lobes. Trends Cogn Sci 12:193-200. https://doi.org/10.1016/j.tics.2008.02.004

Badre D, D'Esposito M (2007) Functional magnetic resonance imaging evidence for a hierarchical organization of the prefrontal cortex. J Cogn Neurosci 19:2082-2099. https://doi.org/10.1162/jocn. 2007.19.12.2082

Badre D, Poldrack RA, Paré-Blagoev EJ, Insler RZ, Wagner AD (2005) Dissociable controlled retrieval and generalized selection mechanisms in ventrolateral prefrontal cortex. Neuron 47:907-918. https://doi.org/10.1016/j.neuron.2005.07.023

Bajada CJ, Jackson RL, Haroon HA, Azadbakht H, Parker GJM, Lambon Ralph MA, Cloutman LL (2017) A graded tractographic parcellation of the temporal lobe. Neuroimage 155:503-512. https://doi.org/10.1016/j.neuroimage.2017.04. 016

Bajada CJ, Trujillo-Barreto NJ, Parker GJMM, Cloutman LL, Lambon Ralph MA (2019) A structural connectivity convergence zone in the ventral and anterior temporal lobes: data-driven evidence from structural imaging. Cortex 120:298-307. https://doi.org/10. 1016/j.cortex.2019.06.014

Bartolomeo P, Seidel Malkinson T (2019) Hemispheric lateralization of attention processes in the human brain. Curr Opin Psychol 29:90-96. https://doi.org/10.1016/j.copsyc.2018.12.023

Behzadi Y, Restom K, Liau J, Liu TT (2007) A component based noise correction method (CompCor) for BOLD and perfusion based fMRI. Neuroimage 37:90-101. https://doi.org/10.1016/j.neuro image.2007.04.042

Bishop SJ, Fossella J, Croucher CJ, Duncan J (2008) COMT val158met genotype affects recruitment of neural mechanisms supporting fluid intelligence. Cereb Cortex 18:2132-2140. https://doi.org/ $10.1093 /$ cercor/bhm 240

Bressler SL, Menon V (2010) Large-scale brain networks in cognition: emerging methods and principles. Trends Cogn Sci 14:277-290. https://doi.org/10.1016/j.tics.2010.04.004

Bright P, Moss H, Tyler LK (2004) Unitary vs multiple semantics: PET studies of word and picture processing. Brain Lang 89:417-432. https://doi.org/10.1016/j.bandl.2004.01.010

Bruyer R, Brysbaert M (2011) Combining speed and accuracy in cognitive psychology: is the Inverse efficiency score (IES) a better dependent variable than the mean reaction time (RT) and the percentage of errors (PE)? Psychologica Belgica 51:5-13

Buckner RL, Krienen FM, Castellanos A, Diaz JC, Yeo BTT (2011) The organization of the human cerebellum estimated by intrinsic functional connectivity. J Neurophysiol 106:2322-2345. https:// doi.org/10.1152/jn.00339.2011

Camilleri JA, Müller VI, Fox P, Laird AR, Hoffstaedter F, Kalenscher T, Eickhoff SB (2018) Definition and characterization of an extended multiple-demand network. Neuroimage 165:138-147. https://doi.org/10.1016/j.neuroimage.2017.10.020

Chiou R, Humphreys GF, Lambon Ralph MA (2020) Bipartite functional fractionation within the default network supports disparate forms of internally oriented cognition. Cereb Cortex 30:54845501. https://doi.org/10.1093/cercor/bhaa130

Coltheart M (1981) The MRC psycholinguistic database. Q J Exp Psychol Sect A 33:497-505. https://doi.org/10.1080/1464074810 8400805 
Corbetta M, Shulman GL (2002) Control of Goal-Directed and Stimulus-Driven Attention in the Brain. Nat Rev Neurosci 3:215-229. https://doi.org/10.1038/nrn755

Corbetta M, Patel G, Shulman GL (2008) The reorienting system of the human brain: from environment to theory of mind. Neuron 58:306-324. https://doi.org/10.1016/j.neuron.2008.04.017

Davey J, Cornelissen PL, Thompson HE, Sonkusare S, Hallam G, Smallwood J, Jefferies E (2015) Automatic and controlled semantic retrieval: TMS reveals distinct contributions of posterior middle temporal gyrus and angular gyrus. J Neurosci: off $\mathbf{J}$ Soc Neurosci 35:15230-15239. https://doi.org/10.1523/JNEUR OSCI.4705-14.2015

Davey J, Thompson HE, Hallam G, Karapanagiotidis T, Murphy C, de Caso I, Krieger-Redwood K, Bernhardt BC, Smallwood J, Jefferies E (2016) Exploring the role of the posterior middle temporal gyrus in semantic cognition: integration of anterior temporal lobe with executive processes. Neuroimage 137:165-177. https://doi. org/10.1016/j.neuroimage.2016.05.051

Ding J, Chen K, Liu H, Huang L, Chen Y, Lv Y, Yang Q, Guo Q, Han Z, Lambon Ralph MA (2020) A unified neurocognitive model of semantics language social behaviour and face recognition in semantic dementia. Nat Commun 11:1-14. https://doi.org/10. 1038/s41467-020-16089-9

Dixon ML, de La Vega A, Mills C, Andrews-Hanna J, Spreng RN, Cole MW, Christoff K (2018) Heterogeneity within the frontoparietal control network and its relationship to the default and dorsal attention networks. Proc Natl Acad Sci 115:E1598-E1607. https://doi.org/10.1073/pnas.1715766115

Duncan J (2001) An adaptive coding model of neural function in prefrontal cortex. Nat Rev Neurosci 2:820-829. https://doi.org/10. $1038 / 35097575$

Duncan J (2010) The multiple-demand (MD) system of the primate brain: mental programs for intelligent behaviour. Trends Cogn Sci 14:172-179. https://doi.org/10.1016/j.tics.2010.01.004

Emch M, von Bastian CC, Koch K (2019) Neural correlates of verbal working memory: An fMRI meta-analysis. Front Hum Neurosci 13:1-17. https://doi.org/10.3389/fnhum.2019.00180

Evans M, Krieger-Redwood K, Gonzalez Alam TRJ, Smallwood J, Jefferies E (2020) Controlled semantic summation correlates with intrinsic connectivity between default mode and control networks. Cortex 129:356-375. https://doi.org/10.1016/j.cortex. 2020.04.032

Fedorenko E, Duncan J, Kanwisher N (2013) Broad domain generality in focal regions of frontal and parietal cortex. Proc Natl Acad Sci USA 110:16616-16621. https://doi.org/10.1073/pnas.13152 35110

Fink GR, Marshall JC, Shah NJ, Weiss PH, Halligan PW, GrosseRuyken M, Ziemons K, Zilles K, Freund HJ (2000) Line bisection judgments implicate right parietal cortex and cerebellum as assessed by fMRI. Neurology 54:1324-1331. https://doi.org/10. 1212/WNL.54.6.1324

Fink GR, Marshall JC, Weiss PH, Zilles K (2001) The neural basis of vertical and horizontal line bisection judgments: an fMRI study of normal volunteers. Neuroimage 14:59-67. https://doi.org/10. 1006/nimg.2001.0819

Fuster JM (2001) The prefrontal cortex - an update: time is of the essence. Neuron 30:319-333. https://doi.org/10.1016/S08966273(01)00285-9

Gao Z, Zheng L, Chiou R, Gouws A, Krieger-Redwood K, Wang X, Varga D, Ralph MAL, Smallwood J, Jefferies E (2021) Distinct and common neural coding of semantic and non-semantic control demands. Neuroimage. https://doi.org/10.1016/j.neuroimage. 2021.118230

Gläscher J, Rudrauf D, Colom R, Paul LK, Tranel D, Damasio H, Adolphs R (2010) Distributed neural system for general intelligence revealed by lesion mapping. Proc Natl Acad Sci USA 107:4705-4709. https://doi.org/10.1073/pnas.0910397107

Glasser MF, Coalson TS, Robinson EC, Hacker CD, Harwell J, Yacoub E, Ugurbil K, Andersson J, Beckmann CF, Jenkinson M, Smith SM, van Essen DC (2016) A multi-modal parcellation of human cerebral cortex. Nature 536:171-178. https://doi.org/10.1038/ nature 18933

Gonzalez Alam T, Murphy C, Smallwood J, Jefferies E (2018) Meaningful inhibition: exploring the role of meaning and modality in response inhibition. Neuroimage 181:108-119. https://doi.org/ 10.1016/j.neuroimage.2018.06.074

Gonzalez Alam TRJ, Karapanagiotidis T, Smallwood J, Jefferies E (2019) Degrees of lateralisation in semantic cognition: evidence from intrinsic connectivity. Neuroimage 202:116089. https://doi. org/10.1016/j.neuroimage.2019.116089

Gonzalez Alam TRJ, Krieger-Redwood K, Evans M, Rice GE, Smallwood J, Jefferies E (2021) Intrinsic connectivity of anterior temporal lobe relates to individual differences in semantic retrieval for landmarks. Cortex 134:76-91. https://doi.org/10. 1016/j.cortex.2020.10.007

Gordon EM, Laumann TO, Adeyemo B, Huckins JF, Kelley WM, Petersen SE (2016) Generation and evaluation of a cortical area parcellation from resting-state correlations. Cereb Cortex 26:288-303. https://doi.org/10.1093/cercor/bhu239

Gotts SJ, Jo HJ, Wallace GL, Saad ZS, Cox RW, Martin A (2013) Two distinct forms of functional lateralization in the human brain. Proc Natl Acad Sci USA 110:E3435-E3444. https://doi. org/10.1073/pnas. 1302581110

Haier RJ, Siegel BV, Nuechterlein KH, Hazlett E, Wu JC, Paek J, Browning HL, Buchsbaum MS (1988) Cortical glucose metabolic rate correlates of abstract reasoning and attention studied with positron emission tomography. Intelligence 12:199-217. https://doi.org/10.1016/0160-2896(88)90016-5

Hearne LJ, Cocchi L, Zalesky A, Mattingley JB (2017) Reconfiguration of brain network architectures between resting-state and complexity-dependent cognitive reasoning. J Neurosci 37:8399-8411. https://doi.org/10.1523/JNEUROSCI.048517.2017

Hobeika L, Diard-Detoeuf C, Garcin B, Levy R, Volle E (2016) General and specialized brain correlates for analogical reasoning: a meta-analysis of functional imaging studies. Hum Brain Mapp 37:1953-1969. https://doi.org/10.1002/hbm.23149

Hoffman P (2018) An individual differences approach to semantic cognition: divergent effects of age on representation, retrieval and selection. Sci Rep 8:1-13. https://doi.org/10.1038/ s41598-018-26569-0

Hoffman P, Jefferies E, Lambon Ralph MA (2010) Ventrolateral prefrontal cortex plays an executive regulation role in comprehension of abstract words: convergent neuropsychological and repetitive TMS evidence. J Neurosci 30:15450-15456. https:// doi.org/10.1523/JNEUROSCI.3783-10.2010

Hong KS, Lee SK, Kim JY, Kim KK, Nam H (2000) Visual working memory revealed by repetitive transcranial magnetic stimulation. J Neurol Sci 181:50-55. https://doi.org/10.1016/S0022-510X(00) 00412-3

Hong SJ, de Wael RV, Bethlehem RAI, Lariviere S, Paquola C, Valk SL, Milham MP, di Martino A, Margulies DS, Smallwood J, Bernhardt BC (2019) Atypical functional connectome hierarchy in autism. Nat Commun 10:1-13. https://doi.org/10.1038/ s41467-019-08944-1

Hugdahl K, Raichle ME, Mitra A, Specht K (2015) On the existence of a generalized non-specific task-dependent network. Front Hum Neurosci 9:430. https://doi.org/10.3389/fnhum.2015.00430

Huntenburg JM, Bazin PL, Margulies DS (2018) Large-scale gradients in human cortical organization. Trends Cogn Sci 22:21-31. https://doi.org/10.1016/j.tics.2017.11.002 
Jackson RL (2021) The neural correlates of semantic control revisited. Neuroimage 224:117444. https://doi.org/10.1016/j.neuroimage. 2020.117444

Jackson RL, Bajada CJ, Rice GE, Cloutman LL, Lambon Ralph MA (2017) An emergent functional parcellation of the temporal cortex. Neuroimage. https://doi.org/10.1016/j.neuroimage.2017.04. 024

Jackson RL, Bajada CJ, Ralph MAL, Cloutman LL (2019) The graded change in connectivity across the ventromedial prefrontal cortex reveals distinct subregions. Cereb Cortex. https://doi.org/10. 1093/cercor/bhz079

Jefferies E (2013) The neural basis of semantic cognition: converging evidence from neuropsychology, neuroimaging and TMS. Cortex 49:611-625. https://doi.org/10.1016/j.cortex.2012.10.008

Jeong SK, Xu Y (2016) The impact of top-down spatial attention on laterality and hemispheric asymmetry in the human parietal cortex. J vis 16:1-21. https://doi.org/10.1167/16.10.2

Jo HJ, Saad ZS, Gotts SJ, Martin A, Cox RW (2012) Quantifying agreement between anatomical and functional interhemispheric correspondences in the resting brain. PLoS One. https://doi.org/10. 1371/journal.pone.0048847

Joliot M, Jobard G, Naveau M, Delcroix N, Petit L, Zago L, Crivello F, Mellet E, Mazoyer B, Tzourio-Mazoyer N (2015) AICHA: An atlas of intrinsic connectivity of homotopic areas. J Neurosci Methods 254:46-59. https://doi.org/10.1016/j.jneumeth. 2015.07.013

Joliot M, Tzourio-Mazoyer N, Mazoyer B (2016) Intra-hemispheric intrinsic connectivity asymmetry and its relationships with handedness and language lateralization. Neuropsychologia 93:437447. https://doi.org/10.1016/j.neuropsychologia.2016.03.013

Karapanagiotidis T, Bernhardt BC, Jefferies E, Smallwood J (2017) Tracking thoughts: exploring the neural architecture of mental time travel during mind-wandering. Neuroimage 147:272-281. https://doi.org/10.1016/j.neuroimage.2016.12.031

Karapanagiotidis T, Vidaurre D, Quinn AJ, Vatansever D, Poerio GL, Turnbull A, Ho NSP, Leech R, Bernhardt BC, Jefferies E, Margulies DS, Nichols TE, Woolrich MW, Smallwood J (2020) The psychological correlates of distinct neural states occurring during wakeful rest. Sci Rep 10:21121. https://doi.org/10.1038/ s41598-020-77336-Z

Karolis VR, Corbetta M, Thiebaut de Schotten M (2019) The architecture of functional lateralisation and its relationship to callosal connectivity in the human brain. Nat Commun 10:1417. https:// doi.org/10.1038/s41467-019-09344-1

Kinsbourne M (1987) Mechanisms of unilateral neglect. In: Jeannerod MBT-AP (ed) Neurophysiological and neuropsychological aspects of spatial neglect. North-Holland, Amsterdam, pp 69-86. https://doi.org/10.1016/S0166-4115(08)61709-4

Knecht S, Deppe M, Dräger B, Bobe L, Lohmann H, Ringelstein EB, Henningsen $H$ (2000) Language lateralization in healthy righthanders. Brain 123:74-81. https://doi.org/10.1093/brain/123.1.74

Koechlin E, Ody C, Kouneiher F (2003) The architecture of cognitive control in the human prefrontal cortex. Science 302:1181-1185. https://doi.org/10.1126/science. 1088545

Krawczyk DC, Hanten G, Wilde EA, Li X, Schnelle KP, Merkley TL, Vasquez AC, Cook LG, McClelland M, Chapman SB, Levin HS (2010a) Deficits in analogical reasoning in adolescents with traumatic brain injury. Front Hum Neurosci 4:1-13. https://doi.org/ 10.3389/fnhum.2010.00062

Krawczyk DC, McClelland MM, Donovan CM, Tillman GD, Maguire MJ (2010b) An fMRI investigation of cognitive stages in reasoning by analogy. Brain Res 1342:63-73. https://doi.org/10.1016/j. brainres.2010.04.039

Krawczyk DC, Michelle McClelland M, Donovan CM (2011) A hierarchy for relational reasoning in the prefrontal cortex. Cortex 47:588-597. https://doi.org/10.1016/j.cortex.2010.04.008
Krieger-Redwood K, Gaskell MG, Lindsay S, Jefferies E (2013) The selective role of premotor cortex in speech perception: a contribution to phoneme judgements but not speech comprehension. J Cogn Neurosci 25:2179-2188. https://doi.org/10.1162/jocn_a_ 00463

Krieger-Redwood K, Teige C, Davey J, Hymers M, Jefferies E (2015) Conceptual control across modalities: graded specialisation for pictures and words in inferior frontal and posterior temporal cortex. Neuropsychologia 76:92-107. https://doi.org/10.1016/j. neuropsychologia.2015.02.030

Lambon Ralph MA, Jefferies E, Patterson K, Rogers TT (2017) The neural and computational bases of semantic cognition. Nat Rev Neurosci 18:42-55. https://doi.org/10.1038/nrn.2016.150

Liang X, Zhao C, Jin X, Jiang Y, Yang L, Chen Y, Gong G (2021) Sex-related human brain asymmetry in hemispheric functional gradients. Neuroimage 229:117761. https://doi.org/10.1016/j. neuroimage.2021.117761

Mancuso L, Costa T, Nani A, Manuello J, Liloia D, Gelmini G, Panero M, Duca S, Cauda F (2019) The homotopic connectivity of the functional brain: a meta-analytic approach. Sci Rep 9:1-19. https://doi.org/10.1038/s41598-019-40188-3

Margulies DS, Ghosh SS, Goulas A, Falkiewicz M, Huntenburg JM, Langs G, Bezgin G, Eickhoff SB, Castellanos FX, Petrides M, Jefferies E, Smallwood J (2016) Situating the default-mode network along a principal gradient of macroscale cortical organization. Proc Natl Acad Sci USA 113:12574-12579. https://doi.org/10.1073/pnas.1608282113

Mckeown B, Strawson WH, Wang HT, Karapanagiotidis T, Vos de Wael R, Benkarim O, Turnbull A, Margulies D, Jefferies E, McCall C, Bernhardt B, Smallwood J (2020) The relationship between individual variation in macroscale functional gradients and distinct aspects of ongoing thought. Neuroimage. https://doi.org/10.1016/j.neuroimage.2020.117072

Medaglia JD, Lynall M-E, Bassett DS (2015) Cognitive network neuroscience. J Cogn Neurosci 27:1471-1491. https://doi.org/ 10.1162/jocn_a_00810

Mikolov T, Sutskever I, Chen K, Corrado GS, Dean J (2013) Distributed representations of words and phrases and their compositionality. In: Burges CJC, Bottou L, Welling M, Ghahramani Z, Weinberger KQ (eds) Advances in neural information processing systems. Curran Associates, Inc., New York, pp 3111-3119

Müller VI, Langner R, Cieslik EC, Rottschy C, Eickhoff SB (2015) Interindividual differences in cognitive flexibility: influence of gray matter volume, functional connectivity and trait impulsivity. Brain Struct Funct 220:2401-2414. https://doi.org/10. 1007/s00429-014-0797-6

Murphy C, Jefferies E, Rueschemeyer S-A, Sormaz M, Wang H, Margulies DS, Smallwood J (2018) Distant from input: evidence of regions within the default mode network supporting perceptually-decoupled and conceptually-guided cognition. Neuroimage 171:393-401. https://doi.org/10.1016/j.neuro image.2018.01.017

Murphy C, Wang HT, Konu D, Lowndes R, Margulies DS, Jefferies E, Smallwood J (2019) Modes of operation: a topographic neural gradient supporting stimulus dependent and independent cognition. Neuroimage 186:487-496. https://doi.org/10.1016/j.neuro image.2018.11.009

Niendam TA, Laird AR, Ray KL, Dean YM, Glahn DC, Carter CS (2012) Meta-analytic evidence for a superordinate cognitive control network subserving diverse executive functions. Cogn Affect Behav Neurosci 12:241-268. https://doi.org/10.3758/ s13415-011-0083-5

Noonan KA, Jefferies E, Visser M, Lambon Ralph MA (2013) Going beyond inferior prefrontal involvement in semantic control: evidence for the additional contribution of dorsal angular gyrus and 
posterior middle temporal cortex. J Cogn Neurosci 25:18241850. https://doi.org/10.1162/jocn

Paquola C, Vos De Wael R, Wagstyl K, Bethlehem RAI, Hong SJ, Seidlitz J, Bullmore ET, Evans AC, Misic B, Margulies DS, Smallwood J, Bernhardt BC (2018) Microstructural and functional gradients are increasingly dissociated in transmodal cortices. bioRxiv. https://doi.org/10.1101/488700

Patterson K, Nestor PJ, Rogers TT (2007) Where do you know what you know? The representation of semantic knowledge in the human brain. Nat Rev Neurosci 8:976-987. https://doi.org/10. $1038 /$ nrn2277

Pereira F, Gershman S, Ritter S, Botvinick M (2016) A comparative evaluation of off-the-shelf distributed semantic representations for modelling behavioural data. Cogn Neuropsychol 33:175-190. https://doi.org/10.1080/02643294.2016.1176907

Petrides M (2005) Lateral prefrontal cortex: architectonic and functional organization. Philos Trans Royal Soc B: Biol Sci 360:781795. https://doi.org/10.1098/rstb.2005.1631

Poerio GL, Sormaz M, Wang HT, Margulies D, Jefferies E, Smallwood J (2017) The role of the default mode network in component processes underlying the wandering mind. Soc Cogn Affect Neurosci 12:1047-1062. https://doi.org/10.1093/scan/nsx041

Popovych OV, Jung K, Manos T, Diaz-Pier S, Hoffstaedter F, Schreiber J, Yeo BTT, Eickhoff SB (2021) Inter-subject and inter-parcellation variability of resting-state whole-brain dynamical modeling. Neuroimage. https://doi.org/10.1016/j.neuroimage.2021.118201

Power JD, Mitra A, Laumann TO, Snyder AZ, Schlaggar BL, Petersen SE (2014) Methods to detect, characterize, and remove motion artifact in resting state fMRI. Neuroimage 84:320-341. https:// doi.org/10.1016/j.neuroimage.2013.08.048

Prabhakaran V, Smith JAL, Desmond JE, Glover GH, Gabrieli JDE (1997) Neural substrates of fluid reasoning: an fMRI study of neocortical activation during performance of the Raven's progressive matrices test. Cogn Psychol 33:43-63. https://doi.org/ 10.1006/cogp.1997.0659

Raemaekers M, Schellekens W, Petridou N, Ramsey NF (2018) Knowing left from right: asymmetric functional connectivity during resting state. Brain Struct Funct 223:1909-1922. https://doi.org/ 10.1007/s00429-017-1604-y

Raven J, Matrices RP, Hill M, Scales V (1994) Manual for Raven's progressive matrices and mill hill vocabulary scales. Advanced Progressive Matrices 41(1):1-48. https://doi.org/10.1006/cogp. 1999.0735

Ren X, Goldhammer F, Moosbrugger H, Schweizer K (2012) How does attention relate to the ability-specific and position-specific components of reasoning measured by APM? Learn Individ Differ 22:1-7. https://doi.org/10.1016/j.lindif.2011.09.009

Ren X, Schweizer K, Xu F (2013) The sources of the relationship between sustained attention and reasoning. Intelligence 41:5158. https://doi.org/10.1016/j.intell.2012.10.006

Rice GE, Hoffman P, Lambon Ralph MA (2015a) Graded specialization within and between the anterior temporal lobes. Ann N Y Acad Sci 1359:84-97. https://doi.org/10.1111/nyas.12951

Rice GE, Lambon Ralph MA, Hoffman P (2015b) The roles of left versus right anterior temporal lobes in conceptual knowledge: an ALE meta-analysis of 97 functional neuroimaging studies. Cereb Cortex 25:4374-4391. https://doi.org/10.1093/cercor/bhv024

Rice GE, Caswell H, Moore P, Ralph MAL, Hoffman P (2018) Revealing the dynamic modulations that underpin a resilient neural network for semantic cognition: an fMRI investigation in patients with anterior temporal lobe resection. Cereb Cortex 28:30043016. https://doi.org/10.1093/cercor/bhy116

Rottschy C, Langner R, Dogan I, Reetz K, Laird AR, Schulz JB, Fox PT, Eickhoff SB (2012) Modelling neural correlates of working memory: a coordinate-based meta-analysis. Neuroimage 60:830-846. https://doi.org/10.1016/j.neuroimage.2011.11.050
Schaefer A, Kong R, Gordon EM, Laumann TO, Zuo X-N, Holmes AJ, Eickhoff SB, Yeo BTT (2018) Local-global parcellation of the human cerebral cortex from intrinsic functional connectivity MRI. Cereb Cortex 28:3095-3114. https://doi.org/10.1093/ cercor/bhx179

Schweizer K, Moosbrugger H (2004) Attention and working memory as predictors of intelligence. Intelligence 32:329-347. https://doi. org/10.1016/j.intell.2004.06.006

Shin M, Jeon H-A (2021) A cortical surface-based meta-analysis of human reasoning. Cerebral Cortex 1-14. https://doi.org/10.1093/ cercor/bhab174

Sormaz M, Murphy C, Wang H, Hymers M, Poerio G, Margulies DS, Sormaz M, Murphy C, Wang H, Hymers M, Karapanagiotidis T, Poerio G (2018) Default mode network can support the level of detail in experience during active task states. Proc Natl Acad Sci 115:9318-9323. https://doi.org/10.1073/pnas.1817966115

Spreng RN, Sepulcre J, Turner GR, Stevens WD, Schacter DL (2013) Intrinsic architecture underlying the relations among the default, dorsal attention, and frontoparietal control networks of the human brain. J Cogn Neurosci 25:74-86. https://doi.org/10. 1162/jocn_a_00281

Stark DE, Margulies DS, Shehzad ZE, Reiss P, Kelly AMC, Uddin LQ, Gee DG, Roy AK, Banich MT, Castellanos FX, Milham MP (2008) Regional variation in interhemispheric coordination of intrinsic hemodynamic fluctuations. J Neurosci: off J Soci Neurosci 28:13754-13764. https://doi.org/10.1523/JNEUROSCI. 4544-08.2008

Szczepanski SM, Konen CS, Kastner S (2010) Mechanisms of spatial attention control in frontal and parietal cortex. J Neurosci 30:148-160. https://doi.org/10.1523/JNEUROSCI.3862-09.2010

Teige C, Cornelissen PL, Mollo G, del Jesus Gonzalez Alam TR, McCarty K, Smallwood J, Jefferies E, del Jesus R, Gonzalez Alam T, McCarty K, Smallwood J, Jefferies E (2019) Dissociations in semantic cognition: oscillatory evidence for opposing effects of semantic control and type of semantic relation in anterior and posterior temporal cortex. Cortex 120:308-325. https:// doi.org/10.1016/j.cortex.2019.07.002

Thiebaut de Schotten M, Dell'Acqua F, Forkel SJ, Simmons A, Vergani F, Murphy DGM, Catani M (2011a) A lateralized brain network for visuospatial attention. Nat Neurosci 14:1245-1246. https:// doi.org/10.1038/nn.2905

Thiebaut de Schotten M, Ffytche DH, Bizzi A, Dell'Acqua F, Allin M, Walshe M, Murray R, Williams SC, Murphy DGM, Catani M (2011b) Atlasing location, asymmetry and inter-subject variability of white matter tracts in the human brain with MR diffusion tractography. Neuroimage 54:49-59. https://doi.org/10.1016/j. neuroimage.2010.07.055

Thiebaut De Schotten M, Urbanski M, Batrancourt B, Levy R, Dubois B, Cerliani L, Volle E (2017) Rostro-caudal architecture of the frontal lobes in humans. Cereb Cortex 27:4033-4047. https://doi. org/10.1093/cercor/bhw215

Tranel D, Grabowski TJ, Lyon J, Damasio H (2005) Naming the same entities from visual or from auditory stimulation engages similar regions of left inferotemporal cortices. J Cogn Neurosci. https:// doi.org/10.1162/0898929055002508

Turnbull A, Wang H-T, Schooler JW, Jefferies E, Margulies DS, Smallwood J (2018) The ebb and flow of attention: between-subject variation in intrinsic connectivity and cognition associated with the dynamics of ongoing experience. Neuroimage 185:286-299. https://doi.org/10.1016/j.neuroimage.2018.09.069

Turnbull A, Karapanagiotidis T, Wang HT, Bernhardt BC, Leech R, Margulies D, Schooler J, Jefferies E, Smallwood J (2020) Reductions in task positive neural systems occur with the passage of time and are associated with changes in ongoing thought. Sci Rep 10:1-10. https://doi.org/10.1038/s41598-020-66698-z 
Valk SL, Xu T, Margulies DS, Masouleh SK, Paquola C, Goulas A, Kochunov P, Smallwood J, Yeo BTT, Bernhardt BC, Eickhoff SB (2020) Shaping brain structure: genetic and phylogenetic axes of macroscale organization of cortical thickness. Sci Adv. https:// doi.org/10.1126/sciadv.abb3417

van Heuven WJB, Mandera P, Keuleers E, Brysbaert M (2014) SUBTLEX-UK: a new and improved word frequency database for British English. Q J Exp Psychol 67:1176-1190. https://doi.org/ 10.1080/17470218.2013.850521

Vandenberghe R, Price C, Wise R, Josephs O, Frackowiak RSJ (1996) Functional anatomy of a common semantic system for words and pictures. Nature 383:254-256. https://doi.org/10.1038/383254a0

Vandierendonck A (2017) A comparison of methods to combine speed and accuracy measures of performance: a rejoinder on the binning procedure. Behav Res Methods 49:653-673. https://doi.org/ 10.3758/s13428-016-0721-5

Vatansever D, Bzdok D, Wang H, Mollo G, Sormaz M, Murphy C, Karapanagiotidis T, Smallwood J, Jefferies E (2017) Varieties of semantic cognition revealed through simultaneous decomposition of intrinsic brain connectivity and behaviour. Neuroimage 158:1-11. https://doi.org/10.1016/j.neuroimage.2017.06.067

Vincent JL, Kahn I, Snyder AZ, Raichle ME, Buckner RL (2008) Evidence for a frontoparietal control system revealed by intrinsic functional connectivity. J Neurophysiol 100:3328-3342. https:// doi.org/10.1152/jn.90355.2008

Visser M, Jefferies E, Lambon Ralph MA (2009) Semantic processing in the anterior temporal lobes: a meta-analysis of the functional neuroimaging literature. J Cogn Neurosci 22:1083-1094. https:// doi.org/10.1162/jocn.2009.21309

Visser M, Lambon Ralph MA (2011) Differential contributions of bilateral ventral anterior temporal lobe and left anterior superior temporal gyrus to semantic processes. J Cogn Neurosci 23:31213131. https://doi.org/10.1162/jocn_a_00007

Vos De Wael R, Larivière S, Caldairou B, Hong SJ, Margulies DS, Jefferies E, Bernasconi A, Smallwood J, Bernasconi N, Bernhardt BC (2018) Anatomical and microstructural determinants of hippocampal subfield functional connectome embedding. Proc Natl Acad Sci USA 115:10154-10159. https://doi.org/10.1073/ pnas. 1803667115

Vos de Wael R, Benkarim O, Paquola C, Lariviere S, Royer J, Tavakol S, Xu T, Hong SJ, Langs G, Valk S, Misic B, Milham M, Margulies D, Smallwood J, Bernhardt BC (2020) BrainSpace: a toolbox for the analysis of macroscale gradients in neuroimaging and connectomics datasets. Commun Biol. https://doi.org/10. 1038/s42003-020-0794-7

Wagner AD, Paré-Blagoev EJ, Clark J, Poldrack RA (2001) Recovering meaning: left prefrontal cortex guides controlled semantic retrieval. Neuron 31:329-338. https://doi.org/10.1016/S08966273(01)00359-2

Wang D, Buckner RL, Liu H (2014) Functional specialization in the human brain estimated by intrinsic hemispheric interaction. $\mathrm{J}$ Neurosci 34:12341-12352. https://doi.org/10.1523/JNEUR OSCI.0787-14.2014

Wang HT, Poerio G, Murphy C, Bzdok D, Jefferies E, Smallwood J (2018a) Dimensions of experience: exploring the heterogeneity of the wandering mind. Psychol Sci 29:56-71. https://doi.org/10. $1177 / 0956797617728727$
Wang X, Bernhardt BC, Karapanagiotidis T, de Caso I, del Gonzalez Alam TRJ, Cotter Z, Smallwood J, Jefferies E (2018b) The structural basis of semantic control: Evidence from individual differences in cortical thickness. Neuroimage 181:480-489. https:// doi.org/10.1016/j.neuroimage.2018.07.044

Wang X, Margulies DS, Smallwood J, Jefferies E (2020) A gradient from long-term memory to novel cognition: transitions through default mode and executive cortex. Neuroimage 220:117074. https://doi.org/10.1016/j.neuroimage.2020.117074

Wechsler D (1955) Wechsler adult intelligence scale. Psychological Corporation, New York

Wertheim J, Ragni M (2018) The neural correlates of relational reasoning: a meta-analysis of 47 functional magnetic resonance studies. J Cogn Neurosci 30:1734-1748. https://doi.org/10.1162/jocn_a_ 01311

Whitfield-Gabrieli S, Nieto-Castanon A (2012) Conn: a functional connectivity toolbox for correlated and anticorrelated brain networks. Brain Connect 2:125-141. https://doi.org/10.1089/brain. 2012.0073

Whitney C, Kirk M, O’Sullivan J, Lambon Ralph MA, Jefferies E (2011) The neural organization of semantic control: TMS evidence for a distributed network in left inferior frontal and posterior middle temporal gyrus. Cereb Cortex 21:1066-1075. https:// doi.org/10.1093/cercor/bhq180

Whitney C, Kirk M, O'Sullivan J, Ralph MAL, Jefferies E, Lambon Ralph MA, Jefferies E (2012) Executive semantic processing is underpinned by a large-scale neural network: revealing the contribution of left prefrontal, posterior temporal, and parietal cortex to controlled retrieval and selection using TMS. J Cogn Neurosci 24:133-147. https://doi.org/10.1162/jocn_a_00123

Wilson M (1988) MRC psycholinguistic database: machine-usable dictionary, version 2. 00. Behav Res Methods Instrum Comput 20:6-10

Yarkoni T, Poldrack RA, Nichols TE, van Essen DC, Wager TD (2011) Large-scale automated synthesis of human functional neuroimaging data. Nat Methods 8:665-670. https://doi.org/10.1038/ nmeth. 1635

Yeo BTT, Krienen FM, Sepulcre J, Sabuncu MR, Lashkari D, Hollinshead M, Roffman JL, Smoller JW, Zollei L, Polimeni JR, Fischl B, Liu H, Buckner RL (2011) The organization of the human cerebral cortex estimated by intrinsic functional connectivity. J Neurophysiol 106:1125-1165. https://doi.org/10.1152/ jn.00338.2011

Zhang M, Wang X, Varga D, Krieger-Redwood K, Margulies D.S, Smallwood J, Jefferies E (2020) Distinct default mode network subsystems show similarities and differences in the effect of task focus across reading and autobiographical memory. bioRxiv. https://doi.org/10.1101/2020.10.03.324947

Zhang M, Varga D, Wang X, Krieger-Redwood K, Gouws A, Smallwood J, Jefferies E (2021) Knowing what you need to know in advance: the neural processes underpinning flexible semantic retrieval of thematic and taxonomic relations. Neuroimage 224:117405. https://doi.org/10.1016/j.neuroimage.2020.117405

Publisher's Note Springer Nature remains neutral with regard to jurisdictional claims in published maps and institutional affiliations. 